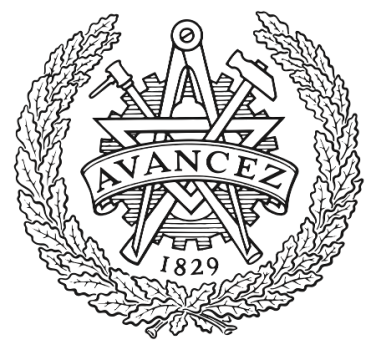

CHALMERS

UNIVERSITY OF TECHNOLOGY

\title{
Minimum Cost Design of Cellular Networks in Rural Areas with UAVs, Optical Rings, Solar Panels and Batteries
}

Downloaded from: https://research.chalmers.se, 2023-04-26 13:49 UTC

Citation for the original published paper (version of record):

Chiaraviglio, L., Amorosi, L., Blefari-Melazzi, N. et al (2019). Minimum Cost Design of Cellular Networks in Rural Areas with UAVs, Optical Rings, Solar Panels and Batteries. IEEE Transactions on Green Communications and Networking. http://dx.doi.org/10.1109/TGCN.2019.2936012

N.B. When citing this work, cite the original published paper. 


\title{
Minimum Cost Design of Cellular Networks in Rural Areas with UAVs, Optical Rings, Solar Panels and Batteries
}

\author{
Luca Chiaraviglio, ${ }^{1,2}$ Lavinia Amorosi, ${ }^{2}$ Nicola Blefari-Melazzi, ${ }^{1,2}$ Paolo Dell'Olmo, ${ }^{3}$ \\ Antonio Lo Mastro, ${ }^{1}$ Carlos Natalino, ${ }^{4}$ Paolo Monti, ${ }^{4}$ \\ 1) EE Department, University of Rome Tor Vergata, email \{name.surname\}@uniroma2.it \\ 2) Consorzio Nazionale Interuniversitario per le Telecomunicazioni, Italy \\ 3) DSS Department, University of Rome Sapienza, email \{name.surname\} @uniroma1.it \\ 4) Chalmers University of Technology, Sweden, email \{carlos.natalino, mpaolo $\} @$ chalmers.se
}

\begin{abstract}
Bringing the cellular connectivity in rural zones is a big challenge, due to the large installation costs that are incurred when a legacy cellular network based on fixed Base Stations (BSs) is deployed. To tackle this aspect, we consider an alternative architecture composed of UAV-based BSs to provide cellular coverage, ground sites to connect the UAVs with the rest of the network, Solar Panels (SPs) and batteries to recharge the UAVs and to power the ground sites, and a ring of optical fiber links to connect the installed sites. We then target the minimization of the installation costs for the considered UAV-based cellular architecture, by taking into account the constraints of UAVs coverage, SPs energy consumption, levels of the batteries and the deployment of the optical ring. After providing the problem formulation, we derive an innovative methodology to ensure that a single ring of installed optical fibers is deployed. Moreover, we propose a new algorithm, called DIARIZE, to practically tackle the problem. Our results, obtained over a set of representative rural scenarios, show that DIARIZE performs very close to the optimal solution, and in general outperforms a reference design based on fixed BSs.
\end{abstract}

Index Terms-CAPEX minimization, cellular networks, UAVbased networks, optical ring installation, renewable energy sources, optimization, algorithms.

\section{INTRODUCTION}

According to the International Telecommunication Union (ITU) [2], the 3G wireless connectivity is largely spread in the world. At the same time, the Internet penetration rates are well above $80 \%$ in the developed countries [3]. However, at least two billion people, mostly living in rural and lowincome areas, are currently experiencing a complete lack of wireless Internet coverage [4]. This is due to the fact that telco operators are not keen to invest in such zones, due to relative low Return On Investment (ROI) rates. On the other hand, urban zones tend to be served by first-class technologies, like the forthcoming $5 \mathrm{G}$ [5]. As a result, the connectivity divide between the people living in urban zones and the ones living in rural areas is going to be further exacerbated in the next years, unless proper counter-measures are put into place.

A preliminary version of this paper appeared in [1].
Rural zones are characterized by very different features compared to urban ones. On one side, in fact, the users are not uniformly spread over the whole rural territory, but they tend to be concentrated in few zones [6]. As a consequence, providing wireless coverage over $100 \%$ of territory would result in a clear waste of radio resources. In addition to this aspect, the electricity grid in rural zones may be not available (and/or not reliable), due to the high installation costs that are faced for bringing electricity to the (few) users [7]. This challenge imposes to exploit renewable energy as a primary source. In addition, the amount of energy that is needed by the network when the renewable energy is not available is drained from a set of batteries [8], which need to ensure the required level of energy requested by the cellular network.

In this context, cellular connectivity provided by BSs mounted on Unmanned Aerial Vehicles (UAVs) is an attractive option to provide the wireless service in rural zones [6]. Compared to a traditional cellular architecture, composed of fixed BSs, UAVs provides several advantages, such as the possibility of covering only portions of territory, good channel conditions w.r.t. the users (typically Line of Sight), and possibility to adapt the coverage service over time by properly scheduling the UAVs missions. Several works in the literature (see e.g., [9], [10], [11], [12], [13], [14], [15], [16], [17], [18], [19], [20]) focus on the management of UAV-based networks, by e.g., scheduling the UAVs missions, selecting the set of areas to be covered, and/or considering UAV energy constraints. However, despite the (important) aspect of management, the minimization of the installation costs for a UAV-based cellular network is also an important (yet overlooked so far) issue. In particular, given a set of areas to be covered, the cellular operator has to face a complex design problem, which involves the cost minimization of the main components that are deployed to realize the UAV-based cellular architecture. This step includes, e.g., the selection of the set of sites that are effectively installed over the territory to provide energy capabilities to the UAVs (and the connection with the rest of the Internet), the dimensioning of each site in terms of SPs and batteries, as well as the selection of the links to interconnect the installed sites through an optical ring. Compared to a traditional cellular architecture, which involves the installation of fixed BSs [8], the design problem in a UAV-based cellular network is a radically different (and 
new) problem, which introduces several constraints - specific of such architecture - that are modeled and analyzed in detail in our work. Specifically, we try to give answers to the following questions:

- Is it possible to formulate the problem of minimizing the installation costs for a cellular architecture composed of ground sites, optical fibers, SPs, batteries and UAVs?

- How many sites need to be installed given a set of areas to be covered, a set of candidate sites, a set of UAVs, and a set of UAV-based coverage constraints?

- Where to place the ground sites to recharge the UAVbased BSs?

- When to recharge a UAV over time? In which site?

- How to dimension each site in terms of number of SPs and number of batteries that are installed?

- How to connect the installed sites through a minimum cost optical ring?

Our innovative contributions can be summarized as follows: i) we provide an optimization framework to model the UAVbased cellular architecture, and to minimize the installation costs, ii) we define a methodology to guarantee the installation of a single ring of optical fibers in the obtained solution, iii) we design an algorithm, called DIARIZE, in order to find a solution even for large instances of the problem, iv) we solve the optimal problem and the DIARIZE algorithm over a set of representative scenarios. Our results prove that the considered solution is able to greatly reduce the costs compared to a classical approach, which assumes the installation of fixed BSs in each area to be covered.

To the best of our knowledge, none of the previous works has tackled a similar (and comprehensive) design problem. Recent works are actually devoted to the exploitation of Renewable Energy Sources (RESs) for traditional cellular networks based on fixed BSs (see e.g., [21], [22], [23], [8], [24]), and not carried on board of the UAVs like in this work. On the other hand, as stated above, there is a pretty vast literature about the management of UAV-based networks, but more focused on the efficient exploitation of the UAVs after that the architecture has been deployed over the territory.

The rest of the paper is organized as follows. We review the related work in Sec. II. Sec. III provides an overview of the UAV-based cellular architecture. The problem formulation and the description of its solution are reported in Sec. IV. Sec. V details the DIARIZE algorithm. Sec. VI includes the description of the scenarios. Results are reported in Sec. VII. Sec. VIII discusses our findings. Finally, Sec. IX concludes this work.

\section{RELATED WORK}

We divide the related work into the following categories: i) cellular networks providing coverage to people living in rural areas, ii) optical rings deployment in 5G fronthaul networks, iii) RESs exploitation in cellular networks, and iv) UAV-based cellular networks. We then provide in the following more details about each category.

\section{A. Cellular Networks for Rural Coverage}

In [7] the authors discuss how the Information and Communication Technology (ICT) could help in the realization of the Sustainable Development Goals (SDGs). In line with them, our work targets economic sustainability, by considering a cost-aware cellular network tailored to rural areas, and environmental sustainability, since the proposed solution allows the full exploitation of sun-based RESs, without the need of consuming brown energy.

The opportunities and challenges for adopting $5 \mathrm{G}$ in order to provide Global Access to the Internet for All (GAIA) are highlighted in [25]. Although their work is mainly focused on an architectural level, the need of reducing the CAPital EXpenditures (CAPEX) costs in deploying the cellular network is recognized as a key enabler. In line with them, also this work targets the reduction of the CAPEX costs. However, we face this goal by developing an optimization framework and an algorithm solution, which are extensively evaluated over different scenarios.

In [6], the authors propose a simple model to compute the monthly subscription fees for users living in rural and low income areas, in order to compensate the CAPEX and OPerating EXpenditures (OPEX) costs of a 5G architecture tailored to serve rural and low-income zones. They consider two distinct possibilities for delivering connectivity to users: i) ultra large cells covering large portions of territory, ii) UAVbased BSs. Results demonstrate that the UAV-based solution is overall able to compensate the CAPEX and OPEX costs. However, the considered approach is based on a simplified model, where the number of devices in terms of SPs, batteries, installed sites, UAVs are not jointly optimized. In addition, the costs for connecting the sites are neglected. In contrast to them, our work explicitly targets the minimization of the CAPEX costs, by proposing an optimization framework, which is able to take into account the different terms of cost. In addition, we model the recharging/covering actions of the UAVs over time, as well as the interconnection of the sites through a ring of fibers.

\section{B. Optical Rings in $5 G$ Fronthaul Networks}

A second taxonomy of works is focused on how to physically interconnect BS sites in a $5 \mathrm{G}$ network. Among the possible solutions, the use of optical Dense Wavelength Division Multiplexing (DWDM) rings brings a number of advantages in terms of cost, energy efficiency, and survivability [26], [27], [28]. More in depth, the authors in [26] discuss the key architectural challenges for the design of a flexible $5 \mathrm{G}$ optical transport infrastructure, by assuming the reduction of the total costs as one of the main drivers. The considered infrastructure is based on the deployment of a hierarchy of access and metro rings connecting the BS sites to the Evolved Packet Core (EPC). Similarly, in [27], the authors assume an architecture based on DWDM rings due to its benefits in terms of high capacity and lower number of fibers (i.e., lower cost) compared to other optical transport options, e.g., architectures based on point-to-point gray interfaces. In addition, the authors in [28] demonstrate how a ring-based DWDM 5G transport 
network brings not only improved performance in terms of cost and energy efficiency but it can inherently guarantee very good survivability levels. Although all these works provide fruitful indications about the cost of deploying $5 \mathrm{G}$ transport networks, they are mainly looking into urban areas, where the BS locations are fixed. On the other hand, in our work, we consider UAV-based BSs and ground sites used to interconnect the UAVs with the rest of the network. This assumption completely changes the problem under exam, since the selection of the sites to be installed is not only based on cost and coverage considerations, but it has also to include UAVs constraints (e.g., maximum flight distance) and the location of the areas that need to be served. In addition, we provide an innovative optimization framework to evaluate the minimization of the CAPEX costs, as well as an algorithm to solve the problem in large scenarios.

\section{Renewable Energy Sources for Cellular Networks}

A third group of works is instead focused on the deployment of sustainable cellular networks exploiting RESs. More in depth, the need of adopting RESs for powering BSs is advocated by [22]. According to [21], powering a heterogeneous cellular network by means of RESs can be a sustainable and economically convenient solution. In [23] the authors consider the adoption of SPs, and dimension them based on: i) the amount of power consumed by the BS, and ii) the amount of power produced by SPs. In addition, they also compute the number of batteries that are needed to absorb the variability of the energy production. In this context, one of the key works is [8], in which the authors propose an algorithm to find the minimum cost combination of SPs and batteries to power a macro Long Term Evolution (LTE) BS. Results demonstrate the feasibility of the proposed solution. Finally, in [24] the authors investigate the impact of quantization for different metrics (e.g., time and energy storage), with the goal of deriving guidelines for the development of realistic models supporting the dimensioning of BSs powered by RESs. Although all these works are surely of interest, as the sustainability is one of the key aspect for the deployment of cellular connectivity in zones not served by the power grid, none of them considers a UAV-based cellular architecture. This assumption, which is instead considered in our work, makes the problem of designing the network radically different from the one considering fixed BSs. For example, the constraints on the UAVs and the scheduling of their recharging actions have a strong impact on the selection of the set of installed sites and their dimensioning in terms of SPs and batteries.

\section{UAV-based Cellular Networks}

The last set of works is instead focused on the analysis of UAV-based cellular networks. More in depth, the problem of limited energy availability from the UAV point of view is raised [29] as one of the issues to be faced. On the other hand, the cellular network planning with UAVs is recognized as one of the key challenges by the extensive tutorial of [30]. In addition, in [31] the authors detail the design and the implementation challenges of an aerial network consisting of
LTE-Advanced (LTE-A) BSs mounted on tethered helikites. Although their work is interesting, it is focused on a different architecture, based on tethered helikite and not UAVs.

Focusing on the technical aspects emerging in UAV-based cellular networks, the authors of [32] present an analytical framework to optimize the altitude of Low-altitude aerial platforms (LAPs) in order to provide maximum radio coverage on the ground. In [9] the authors assume the co-existence between a UAV transmitting data in the downlink and a deviceto-device (D2D) communication network, with the goal of finding the minimum number of stop points that the UAV needs to visit in order to completely cover the area. Results show that there are optimal values for the UAV altitude for which the summation of the rates and the coverage probability are maximized. In [10], the authors assume the deployment of UAVs equipped with directional antennas. In particular, the problem of providing the optimal coverage for a set of UAVs is faced, by also finding the minimum number of UAVs to serve a given geographical area. Eventually, the downlink coverage performance of a network based on Drone Small Cells (DSCs) is investigated by [11]. The authors derive a framework to compute the optimal DSC altitude to guarantee the maximum coverage of the territory, while minimizing the required transmit power. In [12], the authors face the problem of computing the optimal locations of UAVs and the cell boundaries of the coverage areas, showing that the system power efficiency can be greatly improved compared to a classical Voronoi tessellation and fixed UAVs location. In [13] the authors explore the use of UAVs for public safety communications after natural disasters, showing that the throughput coverage and the 5th percentile of spectral efficiency can be improved. The problem of recharging the UAVs on a set of given locations is not investigated.

The problem of minimizing the average network delay by finding the optimal cell partitions of UAVs and terrestrial BSs is faced in [14]. Results show that the proposed approach is able to notably reduce the network delay w.r.t. the classical Signal to Noise Ratio (SNR)-based association. In [15], the authors maximize the minimum downlink throughput for a set of users served by BSs mounted on UAVs, by considering the user scheduling and association, the UAV trajectories, and the BSs transmit power. Moreover, the problem of evaluating the 3D UAV placement considering the backhaul link between the UAV and the backhaul node is investigated by [16]. In addition, the work of [17] is focused on finding the minimum number of UAV-based BSs and their placement in the space so that all the users are served. In [18], the authors show that an optimum UAV height guarantees a beneficial trade-off between path loss and fading, which varies as a function of distance and the elevation angle with respect to the ground terminals. Eventually, the problem of minimizing the number of UAVbased BSs needed to provide wireless coverage for a group of distributed ground terminals, while ensuring coverage of each terminal, is faced by [19]. Finally, the UAV path planning considering geometrically complex environments is faced by [20]. Although all these works prove that there is a great interest in the management UAV-based cellular networks, by considering several important aspects, in this work we focus 


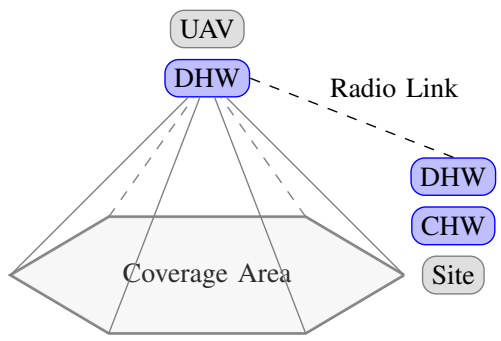

(a) Coverage of an area and radio link between the UAV and the site

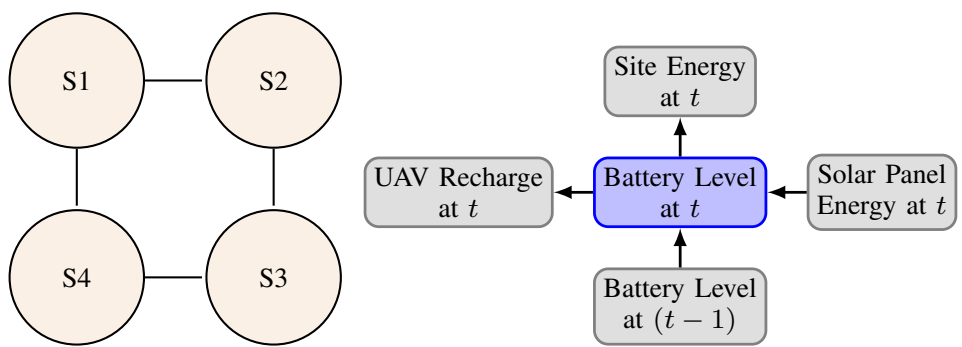

(b) Optical ring deployment to connect the sites (c) Battery level balance on a site

Fig. 1: Main features of the considered UAV-based cellular architecture.

on a different (yet overlooked) problem: the minimization of the installation costs for a UAV-based architecture. In addition, our target is a completely self-sustainable system, where each installed site is solely powered by SPs and batteries. Therefore, the goal of this work is to properly dimension the different elements of the architecture in order to minimize the CAPEX costs. Actually, the outcome of this dimensioning problem can be used as input by approaches that target the efficient management of the UAV-based architecture. For example, the set of installed UAVs, SPs, batteries and sites, which are selected by our solution, can be used as input to further optimization steps during the management of the network, e.g., to improve the actual level of service provided to users and/or to efficiently manage the UAV energy consumption.

\section{UAV-BASEd CEllular ARChitecture}

Fig. 1 summarizes the main components of the UAVbased cellular architecture, which is based on [33], [6]. More in detail, we assume the deployment of softwarized radio Base Stations (BSs), whose functionalities are decomposed in elementary blocks, and run on Commodity Hardware (CHW) or Dedicated Hardware (DHW), as reported in Fig. 1a. In this scenario, the low-level BS functionalities are deployed on the DHW carried by the UAV, while the high-level ones are run on the HW (both DHW and CHW) installed at the ground site. The decoupling between high-level and low-level functionalities allows decreasing the amount of $\mathrm{HW}$ carried by the UAV, and consequently facilitates the moving of the UAVs over the territory. Without loss of generality, quadcopters UAVs are assumed in this work. In addition, an area is covered by a UAV when the UAV reaches the location selected for that particular area (which can be the central one or based on other metrics). In order to ensure the cellular connectivity, the distance between the UAV covering an area and the site at which is connected (through a radio link) has to be lower than a maximum value. This is an essential condition to maintain the connectivity between the low-level functionalities flying on the UAV and the high-level ones placed at the ground site [33].

Focusing then on the ground sites, we assume to control the installation of a subset of sites from a set of candidate ones. In this context, optical fiber links need to be deployed to physically connect the sites. The set of installed sites is then connected by a fiber ring, as sketched in Fig $1 \mathrm{~b}$.
We then target the self-sustainability of the system, by assuming the exploitation of SPs and batteries, which are installed in each ground site. In this way, the energy is only derived from RESs, without the need of exploiting the electricity grid (which we recall is not available/reliable in rural zones). In this context, time is discretized in Time Slots (TSs). In each TS, the battery level of the site is computed as in Fig. 1c, by considering: i) the battery level at previous $\mathrm{TS}$, ii) the energy produced by SPs in the current TS, iii) the energy used to power the ground site (which include, e.g., the CHW and the optical equipment), and iv) the energy used to recharge the UAVs. Finally, in each TS each area is covered by one UAV. On the other hand, a UAV in a specific TS can either: i) cover an area, or ii) recharge itself at a ground site.

Given this architecture, we then target the problem of minimizing the costs of the installed sites, the deployed SPs, the deployed batteries, and the installed optical ring. To this aim, we consider a long-term optimization, where the set of TSs is considered jointly together. To the best of our knowledge, this is the first work targeting such a complex (and innovative) problem. However, the solution of the considered problem is not trivial, due to presence of multiple (and conflicting) constraints, which include: i) the covering of the areas, ii) the maximum distance between each UAV and the ground site at which it is connected, iii) the variation over time of the SPs energy production, iv) the battery levels above a minimum value in all TSs, in order to prevent battery failures [8], v) the maximum number of components (e.g., SPs, batteries, UAVs) that are available, and vi) the correlation between different TSs (e.g., a UAV has to be recharged after covering an area). Consequently, we need to properly model the problem, as detailed in the following section.

\section{PRoblem Formulation}

We first report the notation and the constraints. We then draw the overall picture by presenting the entire problem. Finally, we describe how to solve the optimal problem, by guaranteeing the presence of a single ring in the solution.

\section{A. Notation and constraints}

We initially introduce the following sets: i) $\mathcal{A}$ set of areas to be covered, ii) $\mathcal{S}$ set of candidate sites, iii) $\mathcal{D}$ set of available 
UAVs, iv) $\mathcal{T}$ set of TSs, v) $\mathcal{E}$ set of candidate links among the candidate sites.

For each candidate site $s \in \mathcal{S}$ and for each area $a \in \mathcal{A}$ that needs to be covered, we introduce the matrix $G$, whose elements are Boolean parameters. More in depth, $G_{s, a}$ is equal to 1 if a UAV connected to site $s$ is able to cover area $a$ ( 0 otherwise). The setting of the input matrix $G$ may depend on multiple factors, such as the distance to be covered in order to reach the area, the morphology of the territory, the presence of obstacles, etc. ${ }^{1}$ We then introduce the variables $x_{d, a}^{s}(t) \forall d \in \mathcal{D}, a \in \mathcal{A}, s \in \mathcal{S}, t \in \mathcal{T}$, which take value 1 if UAV $d$ connected to site $s$ covers the area $a$ at TS $t(0$ otherwise). Clearly, at each TS each area has to be covered by a UAV. ${ }^{2}$ We impose this condition through the following constraint:

$$
\sum_{s \in \mathcal{S}} \sum_{d \in \mathcal{D}} G_{s, a} \cdot x_{d, a}^{s}(t)=1, \quad \forall a \in \mathcal{A}, t \in \mathcal{T}
$$

In the following, we introduce the variables $r_{d, s}(t) \forall d \in$ $\mathcal{D}, s \in \mathcal{S}, t \in \mathcal{T}$, which take value 1 if UAV $d$ is recharging on site $s$ at time $t$ ( 0 otherwise). We impose the constraint that each UAV can be recharged in at most one site at each TS:

$$
\sum_{s \in \mathcal{S}} r_{d, s}(t) \leq 1, \quad \forall d \in \mathcal{D}, t \in \mathcal{T}
$$

In the previous constraint, the summation is needed to ensure that a UAV is recharged by at most one ground site at the same time. In other words, we explicitly avoid the (unrealistic) case where a UAV is recharged by multiple ground sites at the same time. In addition, when the UAV has to be recharged, it is physically connected to the batteries of the ground site. During this step, the UAV can not cover any area.

We then impose the installation of a site if a UAV performs a recharge on the site:

$$
r_{d, s}(t) \leq y^{s}, \quad \forall s \in \mathcal{S}, t \in \mathcal{T}, d \in \mathcal{D}
$$

where the variables $y^{s} \forall s \in \mathcal{S}$ take value 1 if candidate site $s$ is installed (0 otherwise).

Similarly to the previous constraint, we impose the installation of a site if a UAV is connected to the site:

$$
x_{d, a}^{s}(t) \leq y^{s}, \quad \forall s \in \mathcal{S}, a \in \mathcal{A}, t \in \mathcal{T}, d \in \mathcal{D}
$$

We then introduce the covering variables $z_{d, a}(t) \forall d \in$ $\mathcal{D}, a \in \mathcal{A}, t \in \mathcal{T}$, which take value 1 if UAV $d$ covers area $a$ at time $t$ ( 0 otherwise). $z_{d, a}(t)$ is then defined as the summation over the sites of the $x_{d, a}^{s}(t)$ variables:

$$
z_{d, a}(t)=\sum_{s \in \mathcal{S}} x_{d, a}^{s}(t), \quad \forall d \in \mathcal{D}, a \in \mathcal{A}, t \in \mathcal{T}
$$

\footnotetext{
${ }^{1}$ In this work, we consider a maximum distance constraint to compute $G_{s, a}$. The investigation of other approaches to compute $G_{s, a}$ is left for future work.

${ }^{2}$ We take into account the worst case scenario, in which the coverage has to be ensured over the whole set of TSs. The relaxation of this constraint to subset of TSs is left for future work.
}

Moreover, we impose the constraint that each UAV in each TS can either covering an area or recharging itself at a ground site:

$$
\sum_{a \in \mathcal{A}} z_{d, a}(t)+\sum_{s \in \mathcal{S}} r_{d, s}(t)=1, \quad \forall d \in \mathcal{D}, t \in \mathcal{T}
$$

In the following, we impose the constraint that, after covering an area at a given TS, the UAV needs to be recharged at a ground site in the following $\mathrm{TS}^{3}$

$$
\sum_{s \in \mathcal{S}} G_{s, a} \cdot r_{d, s}(t) \geq z_{d, a}(t-1), \quad \forall d \in \mathcal{D}, t \in \mathcal{T}, a \in \mathcal{A}(7)
$$

Clearly, the recharge at site $s$ from area $a$ can be done only if the corresponding entry in the $G$ matrix is set to 1 , i.e., if it is possible to reach site $s$ from area $a$.

By jointly imposing constraints (1), (5), (6), (7) we ensure that: i) each area is always covered by one UAV, ii) a UAV can cover at most a single area, iii) when a UAV is recharging it does not cover any area, iv) when a UAV covers an area it is not under recharge, and v) a UAV has to be recharged after performing a coverage action. Therefore, when a UAV is recharging, another one is always providing coverage over an area. Clearly, the number of total UAVs has to be carefully set: if it is too low, then there may be coverage holes. However, this condition can be easily detected by our model, since the problem will trigger an infeasibility warning.

We then introduce the constraints to dimension the SPs and the batteries on each installed site. Let us denote with $E_{\mathrm{R}}^{d}$ the amount of energy needed to recharge UAV $d$. In addition, let us denote with $E_{\mathrm{F}}^{s}$ the fixed energy consumption of site $s$ needed to keep powered on the commodity HW and the optical equipment. We then store the total energy consumption consumed by site $s$ at TS $t$ in the variable $E^{s}(t)$, which is set through the following constraint:

$$
E^{s}(t)=\sum_{d \in D} r_{d, s}(t) \cdot E_{\mathrm{R}}^{d}+y^{s} \cdot E_{\mathrm{F}}^{s}, \quad \forall s \in \mathcal{S}, t \in \mathcal{T}
$$

In the following, we focus on the modelling of the battery levels of each site. We denote with $B_{\text {MAX }}$ and $B_{\text {MIN }}$ the maximum and the minimum battery levels, respectively. We then introduce the variables $N_{\mathrm{B}}^{s}$, which store the number of batteries installed in each site $s$. The total battery levels, expressed by variables $B^{s}(t)$, have to be always between the minimum and maximum values for each TS $s$ and each site $t$, as enforced by the following constraints:

$$
\begin{gathered}
B^{s}(t) \leq B_{\mathrm{MAX}} \cdot N_{\mathrm{B}}^{s}, \quad \forall t \in \mathcal{T}, s \in \mathcal{S} \\
B^{s}(t) \geq B_{\mathrm{MIN}} \cdot N_{\mathrm{B}}^{s}, \quad \forall t \in \mathcal{T}, s \in \mathcal{S}
\end{gathered}
$$

In addition, the battery level $B^{s}(t)$ is computed as the composition of different terms, namely: i) the battery level at previous TS $B^{s}(t-1)$, ii) the energy produced by the installed SPs, which is defined as $E_{\mathrm{SP}}(t) \cdot N_{\mathrm{SP}}^{s}$, where $E_{\mathrm{SP}}(t)$ is an input

\footnotetext{
${ }^{3}$ We assume that each UAV mission last for one TS, by considering (relatively) long TS durations (in the order of an hour). This assumption is reasonable, given the current limited battery capacity of the UAVs. Nevertheless, we believe that the exploitation of more performant UAVs, able to ensure coverage missions over multiple consecutive TSs, is also an interesting step, which we leave for future work.
} 
parameter providing the amount of energy generated by one SP at TS $t$, and $N_{\text {SP }}^{s}$ is a variable storing the number of SPs installed at site $s$, iii) the amount of energy $E^{s}(t)$ consumed by site $s$ at TS $t$, and iv) an eventual surplus of energy $E_{\mathrm{SU}}^{s}(t)$ not used by the site. The relationship between these terms is then expressed by the following constraint:

$$
\begin{aligned}
& B^{s}(t)=B^{s}(t-1)+\quad E_{\mathrm{SP}}(t) \cdot N_{\mathrm{SP}}^{s}-E^{s}(t)-E_{\mathrm{SU}}^{s}(t), \\
& \forall t>1 \in \mathcal{T} \quad \forall s \in \mathcal{S}
\end{aligned}
$$

It is important to note that, by applying the previous constraints, the battery level is bounded by the minimum between Eq. (9) and Eq. (11). In this way, we assume that the surplus of energy $E_{\mathrm{SU}}^{s}(t)$ is either transferred to the grid (if the grid is available) and/or to the users. This alleviates us from the need of dimensioning the sites with a large number of batteries to store the energy surplus.

In the following, we set the initial battery level equal to the maximum one:

$$
B^{s}(1)=B_{\mathrm{MAX}} \cdot N_{\mathrm{B}}^{s}, \quad \forall s \in \mathcal{S}
$$

In addition, we assume that the number of installed batteries and the number of installed SPs have to be lower than the maximum values, which are denoted as $N_{\mathrm{B}}^{\mathrm{MAX}}$ and $N_{\mathrm{SP}}^{\mathrm{MAX}}$, respectively. We impose these conditions through the following constraints:

$$
\begin{aligned}
& N_{\mathrm{B}}^{s} \leq N_{\mathrm{B}}^{\mathrm{MAX}} \cdot y^{s}, \quad \forall s \in \mathcal{S} \\
& N_{\mathrm{SP}}^{s} \leq N_{\mathrm{SP}}^{\mathrm{MAX}} \cdot y^{s}, \quad \forall s \in \mathcal{S}
\end{aligned}
$$

We also impose the fact that a UAV is used if it is recharged or employed for coverage in a given TS:

$$
\begin{aligned}
& z_{d, a}(t) \leq \gamma_{d} \quad \forall d \in \mathcal{D}, a \in \mathcal{A}, t \in \mathcal{T} \\
& r_{d, s}(t) \leq \gamma_{d} \quad \forall d \in \mathcal{D}, s \in \mathcal{S}, t \in \mathcal{T}
\end{aligned}
$$

where $\gamma_{d}$ is a binary variable, taking the value 1 if the UAV $d$ is used in a TS, 0 otherwise.

Eventually, the total number of used UAVs $N_{\mathrm{D}}$ is computed as:

$$
N_{\mathrm{D}}=\sum_{d \in \mathcal{D}} \gamma_{d}
$$

In the following, we consider the constraints relative to the connection of the installed sites through the optical ring. We introduce the variables $f^{s, s^{\prime}} \forall\left(s, s^{\prime}\right) \in \mathcal{E}: s \neq s^{\prime}$, which take value 1 if the optical link between $s$ and $s^{\prime}$ is installed (0 otherwise). We initially impose the constraint that each site $s$ has to be connected by exactly one incoming optical link from another site $s^{\prime}$ and one outgoing link to another site $s^{\prime}$ :

$$
\sum_{s^{\prime} \in \mathcal{S}:\left(s^{\prime}, s\right) \in \mathcal{E}} f^{s^{\prime}, s}+\sum_{s^{\prime} \in \mathcal{S}:\left(s, s^{\prime}\right) \in \mathcal{E}} f^{s, s^{\prime}}=2 y^{s}, \quad \forall s \in \mathcal{S}
$$

However, the previous constraint does not guarantee the presence of a single ring, i.e., two distinct rings not connected by any link are admissible. To avoid this issue, we denote with
$\hat{\mathcal{S}}$ the set of installed sites (i.e., the sites for which $y^{s}=1$ ). We then add the following constraint:

$$
\sum_{s, s^{\prime} \in \overline{\mathcal{S}},\left(s, s^{\prime}\right) \in \mathcal{E}} f^{s, s^{\prime}} \leq|\overline{\mathcal{S}}|-1, \quad \forall \overline{\mathcal{S}} \subset \hat{\mathcal{S}},|\overline{\mathcal{S}}|<|\hat{\mathcal{S}}|
$$

To show a practical example of the previous constraint, let us consider the following set $\hat{\mathcal{S}}=\{1,2,3,4\}$. We only impose Eq. (18), and not Eq. (19). In this scenario, one admissible solution is the following one: $f^{1,2}=1, f^{2,1}=1, f^{3,4}=1$, $f^{4,3}=1$. As a result, two distinct rings, not connected to each other, are installed. What happens by adding Eq. (19)? Let us consider set $\overline{\mathcal{S}}=\{1,2\}$. In this case, constraint (19) is not satisfied, since the left part is $\sum_{s, s^{\prime} \in \overline{\mathcal{S}},\left(s, s^{\prime}\right) \in \mathcal{E}} f^{s, s^{\prime}}=2$, while the right part is $|\overline{\mathcal{S}}|-1=1$. A similar outcome is achieved when set $\overline{\mathcal{S}}=\{3,4\}$ is considered. On the other hand, the ring composed of $f^{1,2}=1, f^{2,3}=1, f^{3,4}=1$, $f^{4,1}=1$ is admissible, since Eq. (19) includes all subsets $\overline{\mathcal{S}}$ whose size is strictly lower than $|\hat{\mathcal{S}}|=\sum_{s} y_{s}=4$. Clearly, to practically introduce this constraint, a run-time approach, which will be presented in Sec. IV-C, has to be pursued.

Finally, we denote the cost of one battery, the cost of one $\mathrm{SP}$ and the cost of one UAV as $C_{\mathrm{B}}, C_{\mathrm{SP}}$, and $C_{\mathrm{D}}$, respectively. For each site $s$ we denote with $C^{s}$ the cost for installing the site $s$ and with $C_{\mathrm{F}}^{s, s^{\prime}}$ the cost for installing the optical fiber link $\left(s, s^{\prime}\right) \in \mathcal{E}$ between sites $s$ and $s^{\prime}$, respectively.

\section{B. Overall Formulation}

The OPTRURALDESIGN problem is then defined as follows:

$$
\begin{array}{r}
\min \sum_{s \in S}(\underbrace{C_{\mathrm{B}} \cdot N_{\mathrm{B}}^{s}}_{\text {Batteries }}+\underbrace{C_{\mathrm{SP}} \cdot N_{\mathrm{SP}}^{s}}_{\mathrm{SPs}}+\underbrace{C^{s} \cdot y^{s}}_{\text {Site Installation }})+ \\
+\underbrace{C_{\mathrm{D}} \cdot N_{\mathrm{D}}}_{\text {UAVs }}+\underbrace{\sum_{\left(s, s^{\prime}\right) \in \mathcal{E}} C_{\mathrm{F}}^{s, s^{\prime}} f^{s, s^{\prime}}}_{\text {Fiber Ring }}
\end{array}
$$

subject to:

$\begin{array}{ll}\text { UAV Coverage Constraints } & (1) \\ \text { UAV Recharge Constraints } & (2),(3) \\ \text { Site Installation Constraints } & (4) \\ \text { UAV Action Constraints } & (5),(6),(7),(15),(16) \\ \text { Site Energy Consumption } & (8) \\ \text { Site Battery Constraints } & (9),(10),(11),(12) \\ \text { Max. SPs/Batteries Constraints } & (13),(14) \\ \text { Number of Used UAVs } & (17) \\ \text { Optical Ring Constraints } & (18),(19) .\end{array}$

under control variables: $y^{s} \in\{0,1\}, x_{d, a}^{s}(t) \in\{0,1\}$, $N_{\mathrm{B}}^{s} \in\left\{0, . ., N_{\mathrm{B}}^{\mathrm{MAX}}\right\}, N_{\mathrm{SP}}^{s} \in\left\{0, . ., N_{\mathrm{SP}}^{\mathrm{MAX}}\right\}, \gamma_{d} \in\{0,1\}$, $f^{s, s^{\prime}} \in\{0,1\}$.

Proposition 1. The OPTRURALDESIGN problem is NPcomplete.

Proof. To prove that the presented problem is NP-complete, we show a reduction from the vertex cover problem known to be NP-complete [34]. A generic instance of the vertex cover problem is a graph $\mathcal{G}=(\mathcal{V}, \overline{\mathcal{E}})$ and an integer $K \leq|\mathcal{V}|$. The 


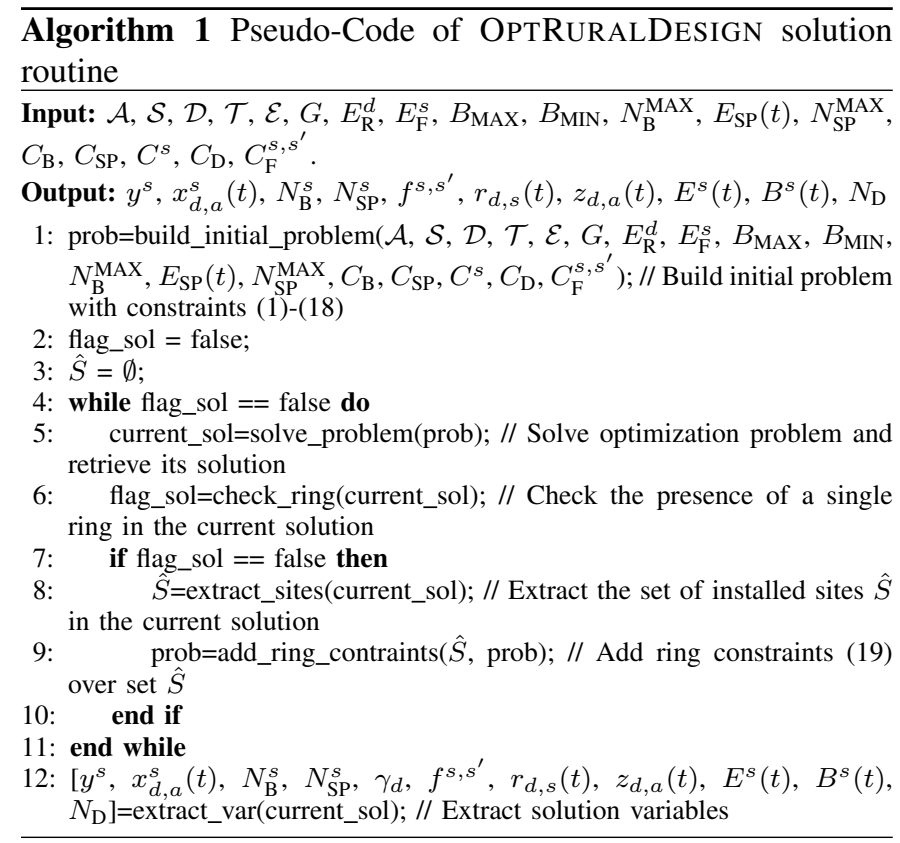

question is then: Is there a vertex cover of size $K$ or less for $\mathcal{G}$, that is a subset $\mathcal{V}^{\prime} \subseteq \mathcal{V}$ such that $\left|\mathcal{V}^{\prime}\right| \leq K$ and for each edge $(i, j) \in \overline{\mathcal{E}}$ at least one of $i$ and $j$ belongs to $\mathcal{V}^{\prime}$ ?

We then consider a particular instance of our problem, in which we assume that $C_{\mathrm{B}}=C_{\mathrm{SP}}=0,|\mathcal{E}|=|\mathcal{T}|=\emptyset$ and $B_{\mathrm{MIN}}=B_{\mathrm{MAX}}=0$. By means of these assumptions the decision version of our problem consists in the following question: Is there a set of recharging sites to be activated which covers all areas and such that the cardinality of this set is less or equal $K$ ?

A generic instance of the vertex cover problem can be directly reduced to this particular case of our problem by associating value 1 to each entry of the matrix $G_{s, a}$ corresponding to each edge $(i, j)$ of the underlying graph. Indeed, for each pair $(i, j)$ of nodes, we can define the element $G_{i, j}$ of the matrix that will be equal to 1 if there is a link between the nodes and 0 otherwise. A solution to our problem with a number of sites less or equal $K$ corresponds to a solution of the vertex cover with $\left|\mathcal{V}^{\prime}\right| \leq K$. This implies that our problem is NP-complete.

\section{Solving the OptRURALDESIGN Problem}

One of the most challenging aspects of the OPTRURALDESIGN problem is how to practically deal with the constraint (19), which ensures that a single ring, passing for all the installed sites, is deployed. This constraint requires: i) the set of installed sites $\hat{\mathcal{S}}$, ii) all the possible subsets of sites $\overline{\mathcal{S}} \in \hat{\mathcal{S}}$, excluding $\hat{\mathcal{S}}$ itself. Clearly, the number of constraints grows exponentially with $\hat{\mathcal{S}}$. In addition, the set $\hat{\mathcal{S}}$ is selected by the problem, and cannot be known in advance. Therefore, we have developed a methodology to solve the OPTRURALDESIGN problem including Eq. (19), by taking inspiration from the subtour elimination constraints of the Traveling Salesman Problem (TSP) [35], but providing an innovative way of solution. Alg. 1 reports the pseudocode of our solution routine. More in detail, the required input parameters are the input sets $\mathcal{A}, \mathcal{S}, \mathcal{D}, \mathcal{T}, \mathcal{E}$, the coverage matrix $G_{s, a}$, the energy parameters $E_{\mathrm{R}}^{d}, E_{\mathrm{F}}^{s}$, the battery parameters $B_{\mathrm{MAX}}, B_{\mathrm{MIN}}$, and $N_{\mathrm{B}}^{\mathrm{MAX}}$, the SP parameters $E_{\mathrm{SP}}(t)$ and $N_{\mathrm{SP}}^{\mathrm{MAX}}$, the number of UAVs $N_{\mathrm{D}}$, and the cost parameters $C_{\mathrm{B}}, C_{\mathrm{SP}}, C^{s}, C_{\mathrm{D}}, C_{\mathrm{F}}^{s, s^{\prime}}$. The routine then produces as output the setting for the control variables $y^{s}, x_{d, a}^{s}(t), N_{\mathrm{B}}^{s}, N_{\mathrm{SP}}^{s}$, $\gamma_{d}, f^{s, s^{\prime}}$, as well as for the secondary variables $r_{d, s}^{t}, z_{d, a}(t)$, $E^{s}(t), B^{s}(t)$.

Initially, the problem is built, by considering the input parameters passed to the routine and constraints (1)-(18) (line 1). A solution flag is then set to false (line 2 ) and $\hat{\mathcal{S}}$ is initialized to the empty set (line 3 ). In the following, the problem iterates over a while cycle which depends on the value of the flag (line 4). For each iteration, the problem is solved (line 5), and a check on the obtained ring solution is performed (line 6). If the solution includes more than one ring, the flag is set to false, the set of current installed sites $\hat{\mathcal{S}}$ is extracted (line 8), and the ring constraints of Eq. (19) are added for $\hat{\mathcal{S}}$ to the problem (line 9). Consequently, the routine passes to the next iteration. Otherwise, if the current solution includes only one ring, the flag is set to true, the while cycle is terminated (line 4), and the output variables are produced as output (line 12).

\section{DIARIZE ALGORITHM}

In order to solve the presented problem also for large instances, we propose a new algorithm, called DESIGN ALGORITHM FOR RURAL ZONES (DIARIZE), which is sketched in Alg. 2. DIARIZE requires as input the same parameters of the OPTRURALDESIGN model, plus the matrix of distances between each candidate site and each area. DIARIZE then produces as output the set of installed sites $y^{s}$, the set of installed optical links $f^{s, s^{\prime}}$, the association between each area and each site of each UAV at each TS $x_{d, a}^{s}(t)$, the scheduling of the recharging/covering actions $r_{d, s}(t)$ and $z_{d, a}(t)$, the number of installed batteries $N_{\mathrm{B}}^{s}$ and installed SPs $N_{\mathrm{SP}}^{s}$, the energy consumed by each site in each TS $E^{s}(t)$, the battery level for each site in each TS $B^{s}(t)$ and the UAVs usage variables $\gamma_{d}$.

In order to retrieve the solution, DIARIZE applies a divide et impera approach, where the complex problem of optimal design is split into the following routines: i) selection of the pool of installed sites through an unsupervised approach based on the k-medoids algorithm (line 1), ii) pruning of the pool in order to retrieve only the solutions ensuring coverage (line $3-5)$, iii) computation of the optical ring to connect the set of installed sites of each solution in the pool (lines 6-7), iv) site dimensioning in terms of SPs, batteries and assigned UAVs for each solution (line 8), v) selection of the minimum cost solution in the pool and related output parameters (lines 917). In the following subsections, each routine is presented with more detail. Finally, the computational complexity of the entire algorithm is discussed.

\section{A. Selection of the pool of installed sites}

During this step, DIARIZE finds a pool of solutions in terms of installed sites by applying the k-medoids algorithm, 


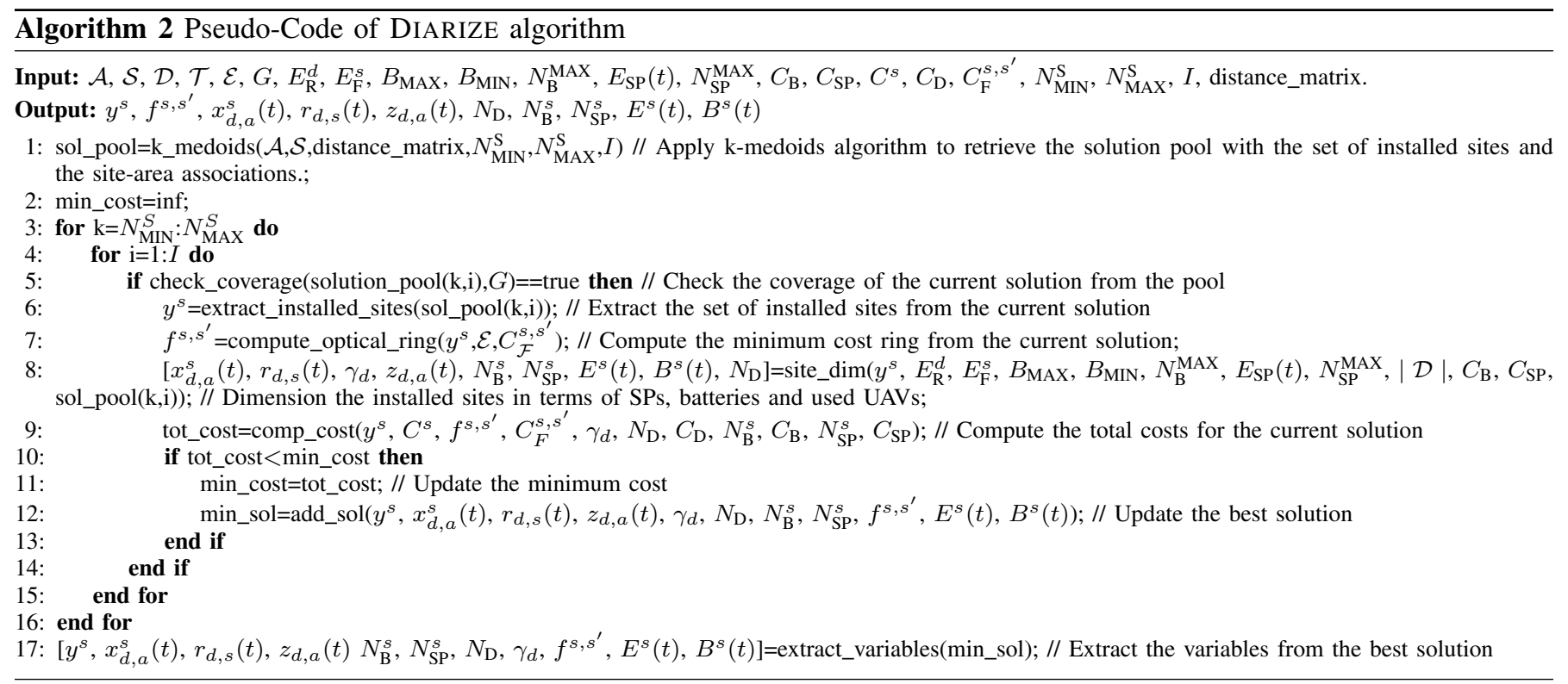

a clustering algorithm widely used in the literature [36]. We refer the reader to [36] for a detailed description of the kmedoids routine, while here we report the main steps, which are tailored to the considered problem. The routine requires as input the set of candidate sites $\mathcal{S}$, the set of areas $\mathcal{A}$ and the matrix of the distances between each site and the areas. In addition, the minimum and the maximum number of installed sites for each solution ( $N_{\mathrm{MIN}}^{\mathrm{S}}$ and $N_{\mathrm{MAX}}^{\mathrm{S}}$, respectively) have to be specified. Finally, a parameter, denoted with $I$, is used to limit the number of cycles of the k-medoids routine.

The k-medoids algorithm is then iterated $\forall k: N_{\text {MIN }}^{\mathrm{S}}<k<$ $N_{\text {MAX }}^{\mathrm{S}}$ and $\forall i: 1 \leq i \leq I$. During each iteration, the algorithm performs the following steps: i) random selection of a set of $k$ installed sites from the $\mathcal{S}$ set, ii) association of each area to the closest installed site, iii) computation of the cost metric $C$ for each site and each connected area, iv) selection of a random site out of the $\mathcal{S}$ set (excluding the sites already selected during i)), v) permutation of the site selected in iv) and a random site found at step i), vi) computation of the cost metric $C^{\prime}$ after the permutation, vii) if $C^{\prime}<C$ holds then the permutation is kept and the routine goes to step iv), otherwise it is reverted and the algorithm switches to the next iteration.

Focusing on the cost function, we adopt the summation of the distances between each site and the served areas. In this way, the obtained solutions should select medoids whose distance from the served areas is included in the cost function, and hence it tends to be limited. However, we point out that other metrics, including e.g., the cost of the sites $C^{s}$, may be introduced. In any case, at the end of the routine a pool of solutions, each of them including a subset of installed sites, is returned.

\section{B. Pruning of the solutions pool}

In the following, DIARIZE selects only the solutions that are feasible from a coverage point of view, by running the check_coverage function of Alg. 2 (line 5) on each solution in the pool. To this aim, a compatibility check against the $G$ matrix is performed, by considering a constraint similar to Eq. (1). In particular, let us denote with $L_{s, a}$ a matrix whose elements take value 1 if area $a$ has been assigned to site $s$ in the current solution of the pool, 0 otherwise. For each area $a$, a check on the term $\prod_{a} \sum_{s} G_{s, a} L_{s, a}$ is then performed: if the previous term is equal to zero, there is at least one area which is not covered, and hence the current solution is not further considered by the following steps of the algorithm, since it is not feasible. Otherwise, the algorithm passes to the next step.

\section{Computation of the optical ring}

In the following, we perform the computation of the minimum cost optical ring for connecting the installed sites, which is performed inside the compute_optical_ring function at line 7 of Alg. 2. This routine is performed on each feasible solution of the pool. Actually, computing the minimum cost ring by enumerating all the possible ones may be a challenging step, and therefore we rely on a practical approach. More in detail, an initial site is selected to be the starting node of the link of the optical ring. In our case, we adopt the first site id in lexicographical order. Let us denote this site as $s$. The cost for connecting the current site $s$ to all the other installed ones is then evaluated, and the site with the minimum cost is selected, i.e., $\arg _{s^{\prime}} \min _{\left(s, s^{\prime}\right) \in \mathcal{E}} C_{F}^{s, s^{\prime}}, \forall s^{\prime}: y^{s^{\prime}}>0$. Consequently, the corresponding link is installed $\left(f^{s, s^{\prime}}=1\right)$, and the procedure is repeated by considering $s^{\prime}$ as starting node of the next link. Finally, the last node is connected with the initial one to close the ring.

\section{Site Dimensioning}

The goal of the following phase is to properly dimension the installed sites of each solution in terms of SPs, batteries and used UAVs. This step is done in the site_dim function (line 8 of Alg. 2), whose pseudo-code is expanded in Alg. 3. The function requires as input the set of installed sites $y^{s}$, the energy parameters $E_{\mathrm{R}}^{d}$ and $E_{\mathrm{F}}^{s}$, the SPs parameters $E_{\mathrm{SP}}(t)$, 


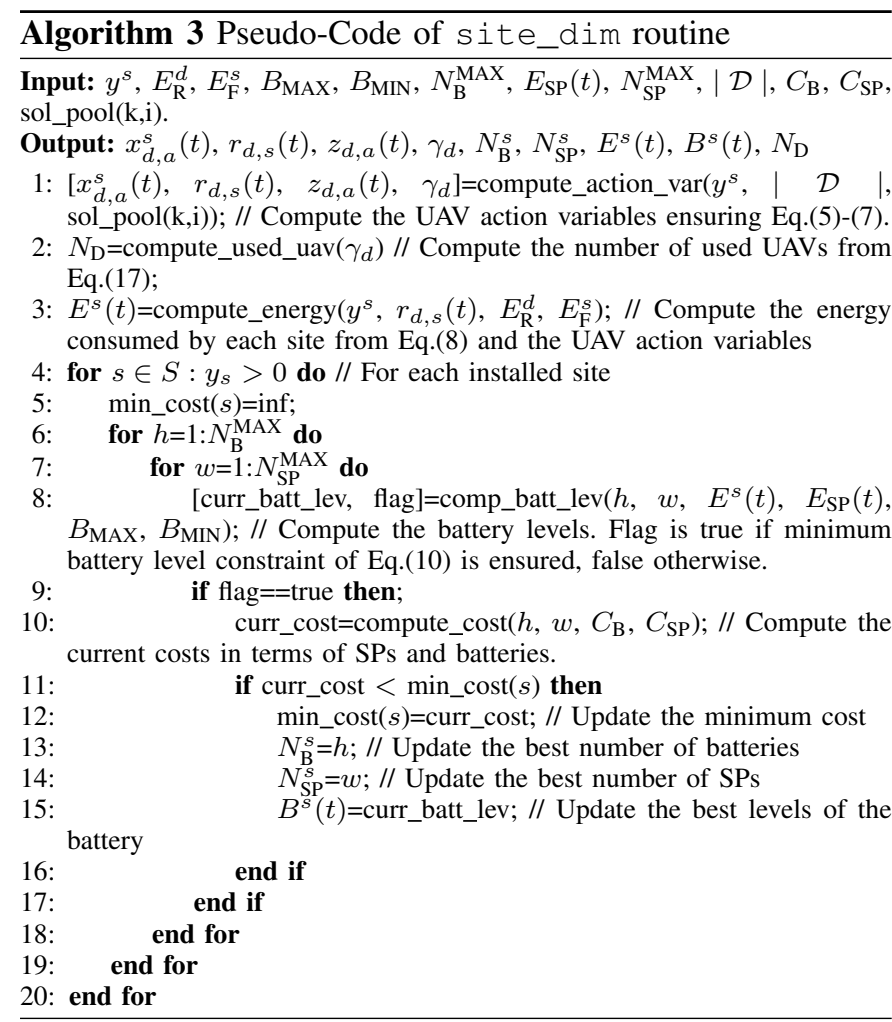

$N_{\mathrm{SP}}^{\mathrm{MAX}}$, the battery parameters $B_{\mathrm{MAX}}, B_{\mathrm{MIN}}, N_{\mathrm{B}}^{\mathrm{MAX}}$, the number of UAVs $|\mathcal{D}|$, the cost parameters $C_{\mathrm{B}}$ and $C_{\mathrm{SP}}$, and the association of areas to sites for the current solution, which is included in the sol_pool $(\mathrm{k}, \mathrm{i})$ structure. The function then produces as output the setting of the action variables $x_{d, a}^{s}(t), r_{d, s}(t)$ and $z_{d, a}(t)$, the setting of the UAVs usage variables $\gamma_{d}$, the number of installed SPs $N_{\mathrm{SP}}^{s}$ and batteries $N_{\mathrm{B}}^{s}$ for each site, the site energy consumption $E^{s}(t)$, the battery levels $B^{s}(t)$ and the number of used UAVs $N_{\mathrm{D}}$.

Initially (line 1 ), the action variables $x_{d, a}^{s}(t), r_{d, s}(t), z_{d, a}(t)$ and UAVs variables $\gamma_{d}$ are computed, by assuming that each UAV always recharge itself at the same site during the considered set of TSs and by ensuring Eq. (5)-(7). In the following (line 2), the total number of used UAVs is computed, by applying Eq.(17). Then (line 3), the routine computes the total energy consumed by each site in each TS, by solving Eq. (8). A brute force approach is then applied to find the best combinations of SPs and batteries limiting the installation costs of these devices while ensuring the battery constraints (lines 4-20). More in depth, the installed sites are sequentially evaluated (line 4). For each installed site, all the possible combinations of $h$ SPs and $w$ batteries are tested (lines 6-7). In the following, the battery levels are computed by the comp_batt_lev routine (line 8 ). Inside this function, the battery level in each TS is computed as $B^{s}(t)=\min \left[B_{\mathrm{MAX}} \cdot w,\left(B^{s}(t-1)+E_{\mathrm{SP}}(t) \cdot h-E^{s}(t)\right)\right]$. In the following, if the constraint of the minimum battery level is not satisfied (i.e., $B^{s}(t)<B_{\mathrm{MIN}} \cdot w$ ), an output flag is set to false, and the current configuration is discarded. Otherwise, if the constraint is satisfied (i.e., $B^{s}(t) \geq B_{\mathrm{MIN}} \cdot w$ ), the output flag is set to true, the cost of the current configuration is evaluated (line 10), and eventually stored if lower than the

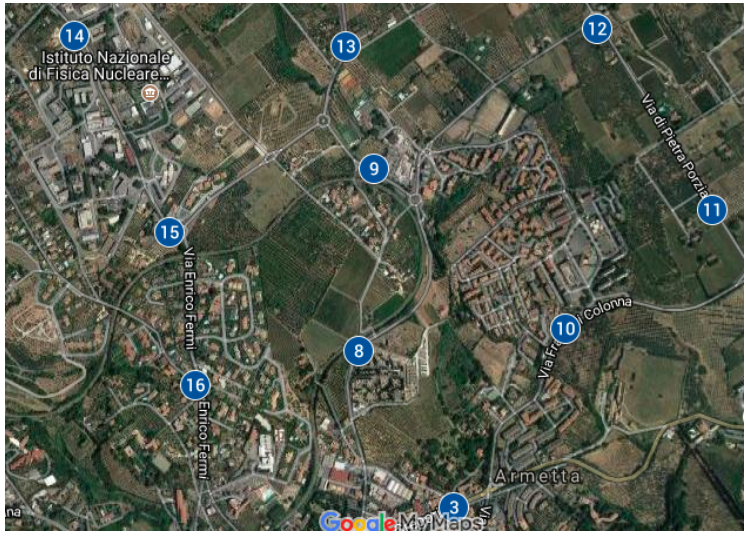

(a) Frascati Small Scenario

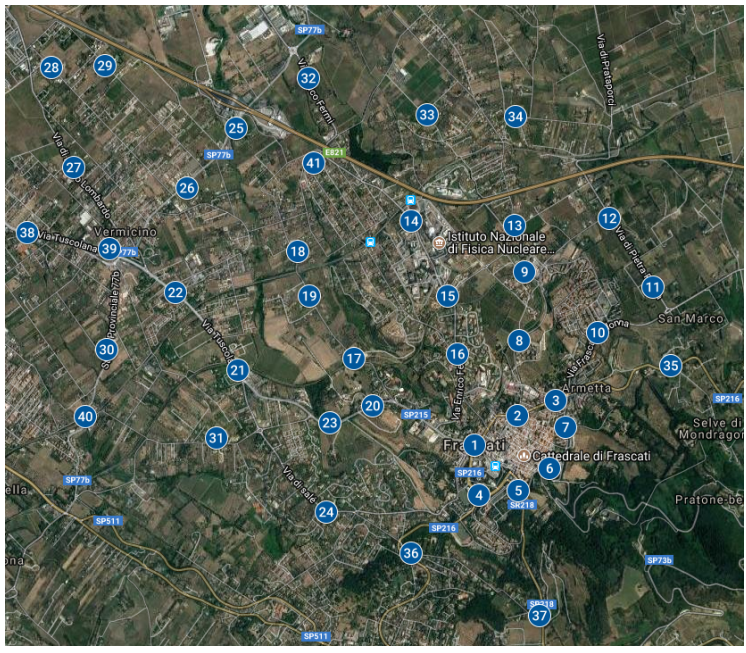

(b) Frascati Big Scenario

Fig. 2: Positions of the candidate sites and the centers of the areas (source of terrain map: Google Earth).

current minimum one (lines 11-15).

\section{E. Selection of the minimum cost solution}

The last step of the DIARIZE algorithm is the selection of the best feasible configuration reducing the whole costs (lines 9-13 of Alg. 2). We recall that the searching of the feasible configurations is done by iterating over the number of medoids $k$, which is bounded by the input parameters $N_{\mathrm{MIN}}^{\mathrm{S}}$ and $N_{\mathrm{MAX}}^{\mathrm{S}}$, and the number of cycles $i$, which is instead bounded by input parameter $I$. Finally, the variables associated to the best configuration are retrieved (line 17 of Alg. 2).

\section{F. Computational Complexity}

We analyze the time complexity of DIARIZE, by first evaluating the complexity of its routines in isolation. The $\mathrm{k}$ _medoids routine depends on the implementation of the $\mathrm{k}$ medoids algorithm. In particular, by preliminary ordering the area-site pairs by increasing distance, it is possible to reach a time complexity of $\mathcal{O}(|\mathcal{S}| \times|\mathcal{A}| \log (|\mathcal{S}| \times|\mathcal{A}|$ )$\left.+N_{\text {MAX }}^{\mathrm{S}} \times I \times|\mathcal{S}| \times|\mathcal{A}|\right)$. Given the current solution, the complexity of the extract_installed_sites routine is 
TABLE I: Parameters of the scenarios

\begin{tabular}{|c|c|c|c|c|}
\hline Parameter & Description & \multicolumn{2}{|c|}{ Scenario } & Appears in Eq./Reference \\
\hline $\mathcal{S}$ & Set of candidate sites & Fig. $2 \mathrm{a},|\mathcal{S}|=10$ & Fig. $2 \mathrm{~b},|\mathcal{S}|=41$ & $\begin{array}{l}(1),(2),(4),(5),(6),(7),(8),(9),(10),(11),(12),(13), \\
(14),(16),(18),(19),(20)\end{array}$ \\
\hline $\mathcal{A}$ & Set of the areas & Fig. 2a, $|\mathcal{A}|=10$ & Fig. $2 \mathrm{~b},|\mathcal{A}|=41$ & $(1),(4),(5),(6),(7),(15)$ \\
\hline $\mathcal{D}$ & Set of UAVs & $|\mathcal{D}|=20$ & $|\mathcal{D}|=82$ & $(1),(2),(3),(4),(5),(6),(7),(8),(15),(16),(17),(20)$ \\
\hline $\mathcal{T}$ & Set of TSs & $|\mathcal{T}|=720$ & $|\mathcal{T}|=\{720\}$ & $(1),(2),(3),(4),(5),(6),(7),(8),(9),(10),(11),(12),(15),(16)$ \\
\hline$G_{s, a}$ & Coverage matrix & $\begin{array}{l}\text { From Fig. 2a } \\
\text { with max. dista }\end{array}$ & $\begin{array}{l}\text { From Fig. (2b) } \\
\text { nce of } 900[\mathrm{~m}]\end{array}$ & $(1),(7)$ \\
\hline $\mathcal{E}$ & Set of candidate links & $|\mathcal{E}|=90$ & $|\mathcal{E}|=1640$ & $(18),(19),(20)$ \\
\hline$E_{\mathrm{R}}^{d}$ & UAV Rec. Energy & \multicolumn{2}{|c|}{$200[\mathrm{Wh}]$} & $(8) /[37]$ \\
\hline$E_{\mathrm{F}}^{S}$ & Site Energy & \multicolumn{2}{|c|}{$1000[\mathrm{Wh}]$} & $(8) /[6]$ \\
\hline$B_{\mathrm{MAX}}$ & Max. Battery Level & \multicolumn{2}{|c|}{$2400[\mathrm{Wh}]$} & $(9) /[8]$ \\
\hline$B_{\mathrm{MIN}}$ & Min. Battery Level & \multicolumn{2}{|c|}{$720[\mathrm{Wh}]$} & $(10) /[8]$ \\
\hline$E_{\mathrm{SP}}(t)$ & Produced SP Energy & \multicolumn{2}{|c|}{ Historical data } & $(11) /[38]$ \\
\hline$C^{s}$ & Site Installation Cost & \multicolumn{2}{|c|}{$40000[€]$} & $(20) /[6]$ \\
\hline$C_{\mathrm{D}}$ & UAV Cost & \multicolumn{2}{|c|}{$4300[€]$} & $(20) /[6]$ \\
\hline$C_{\mathrm{SP}}$ & SP Cost & \multirow{2}{*}{\multicolumn{2}{|c|}{$\begin{array}{l}800[€] \\
150[€]\end{array}$}} & $(20) /[8]$ \\
\hline$C_{\mathrm{B}}$ & Battery Cost & & & $(20) /[8]$ \\
\hline$C_{\mathrm{F}}^{s, s^{\prime}}$ & Fiber Cost & Fig.3a & Fig. $3 b$ & (20) / Tab. II and cost computation based on distance \\
\hline$N_{\mathrm{B}}^{\mathrm{MAX}}$ & Max. Batteries & 50 & 30 & $(13)$ \\
\hline$N_{\mathrm{SP}}^{M A X}$ & Max. SPs & 100 & 30 & (14) \\
\hline
\end{tabular}

TABLE II: Fiber cost parameters

\begin{tabular}{|c|c|l|}
\hline Type & Cost $[€ \times 1000 / \mathrm{km}]$ & Sites \\
\hline Historical Center & 300 & $\{1,2,3,4,5,6,7\}$ \\
\hline Countryside & 100 & $\{8,9,11,12,13,17,18$, \\
& & $19,24,28,29,31,33,34\}$ \\
\hline Main roads & 50 & $\{10,14,15,16,20,21,22$, \\
& & $23,25,26,27,30,32,35$, \\
& & $36,37,38,39,40,41\}$ \\
\hline
\end{tabular}

$\mathcal{O}(|\mathcal{S}|)$. On the other hand, the compute_optical_ring function requires to find, for each installed site, the minimum cost fiber, resulting then in a worst-case complexity of $\mathcal{O}(|\mathcal{S}|$ $\times|\mathcal{E}|)$. Moreover, the site_dim function has a complexity of $\mathcal{O}\left(|\mathcal{S}| \times|\mathcal{T}|\left(|\mathcal{D}| \times|\mathcal{A}|+N_{\mathrm{B}}^{\mathrm{MAX}} \times N_{\mathrm{SP}}^{\mathrm{MAX}}\right)\right)$. This is due to the fact that: i) the setting of the action variable $x_{d, a}^{s}(t)$ requires at most $|\mathcal{S}| \times|\mathcal{T}| \times|\mathcal{D}| \times|\mathcal{A}|$ iterations, ii) the computation of the energy consumed in each site $E^{s}(t)$ has a time complexity of $|\mathcal{S}| \times|\mathcal{T}| \times|\mathcal{D}|$, iii) the following steps of the routine require $|\mathcal{S}| \times|\mathcal{T}| \times N_{\mathrm{B}}^{\mathrm{MAX}} \times N_{\mathrm{SP}}^{\mathrm{MAX}}$ iterations in the worst case.

The overall time complexity of DIARIZE is $\mathcal{O}(|\mathcal{S}| \times \mid$ $\mathcal{A}|\log | \mathcal{S}|\times| \mathcal{A}\left|+N_{\text {MAX }}^{\mathrm{S}} \times I \times\right| \mathcal{S} \mid(|\mathcal{E}|+|\mathcal{T}|(\mid$ $\left.\left.\left.\mathcal{D}|\times| \mathcal{A} \mid+N_{\mathrm{B}}^{\mathrm{MAX}} \cdot N_{\mathrm{SP}}^{\mathrm{MAX}}\right)\right)\right)$. Although the complexity may appear non-negligible at a first glance, we point out that it is mainly governed by the input parameters $N_{\mathrm{MAX}}^{\mathrm{S}}, I, N_{\mathrm{B}}^{\mathrm{MAX}}$, $N_{\mathrm{SP}}^{\mathrm{MAX}}$, which may be set in accordance to the considered scenario. We will discuss the setting of these parameters in more detail in Sec. VI and in Sec. VII.

\section{DESCRIPTION OF THE SCENARIOS}

We consider a rural area in Italy, spanning over the territory of Frascati, a small town located in the Roman countryside. We then select two representative portions of territory, called "Frascati Small" and "Frascati Big", as considered scenarios. Fig. 2 reports the locations of the candidate sites and the centers of the areas as pins in the maps for each scenario. The sites and the centers of the areas are chosen by adhering to the following guidelines: i) placing the pins sufficiently sparse over the territory, ii) avoiding to put the pins directly over sensible places, such as buildings, iii) exploiting as much as possible the proximity to roads in order to not introduce additional site costs (e.g, road construction to reach the site). The two scenarios differ mainly in the cardinality of the number and sites and the number of areas, which is reported in Tab. I. In this way, we expect that it is feasible to compare both OPTRURALDESIGN and DIARIZE in the Frascati Small scenario. On the other hand, the Frascati Big scenario includes a larger portion of territory, and therefore a much more complex problem, which we expect to be solely solvable by DIARIZE. Clearly, we point out that the set of areas is an input to our problem. This set has to be carefully selected, in order e.g. to avoid interference issues among the UAVs (in case the UAVs are using the same frequency band). Therefore, depending on its needs, an operator can choose a proper set of input areas, which is passed as input to our problem.

We then discuss in more detail the remaining parameters reported in Tab. I. The number of UAVs is set equal to the double of the number of areas, in order to guarantee the feasibility of the proposed problem in terms of areas to be covered and recharge of the UAVs. In addition, we assume a TS duration of $1[\mathrm{~h}]$ for a total number of TSs covering 1 [month] for both scenarios. We remind that, in our work, each UAV mission starts and ends in one TS. The considered TS duration is in line with the battery capabilities of currently available quadcopters UAVs (see e.g., [39]). Focusing then on the coverage matrix $G$, it is computed from the positions of sites and centers of the areas of Fig. 2a- 2 b, by imposing a maximum distance of $900[\mathrm{~m}]$. By adopting this setting, the time to reach an area from a site (and vice-versa) is much lower compared to the TS duration. For example, by assuming a speed of $10[\mathrm{~m} / \mathrm{s}]$ for the UAV, the traveling time between the site and the area would take less than 2 [minutes] in the worst case. In addition, we remark the fact that the distance 
between the UAV covering an area and the site at which is connected should not be too high, in order to guarantee an adequate quality of the radio link between the UAV and the site.

In the following, we consider the cost of fibers. To this aim, we initially divide the candidate sites into the following categories: i) historical center of the town, ii) main roads, and iii) countryside. We then associate a cost per $\mathrm{km}$ for each category, based on the feedback from a telecom operator. Tab. II reports the obtained fiber parameters. Clearly, the fiber cost is relatively high when the site is located in the historical center. In addition, deploying a fiber in the countryside has also a nonnegligible cost, mainly due to the need of building/enlarging roads to reach the site, as well as overcoming obstacles such as rivers and streams. Eventually, the fiber cost is lower than in the other cases if the site is deployed on a side of a main road, thus exploiting the road infrastructure which is already available. Given the fiber cost per site, we compute the total cost to deploy the fiber between each node pair $\left(s, s^{\prime}\right)$, which we recall is denoted as $C_{\mathrm{F}}^{s, s^{\prime}}$. To this aim, we assume that $C_{\mathrm{F}}^{s, s^{\prime}}$ is computed as the average cost of the two endpoints of the pair, multiplied by their Euclidean distance. ${ }^{4}$ The resulting matrices reporting the fiber cost are reported in Fig. 3 for the two scenarios.

We then consider the parameters related to energy. We set $E_{\mathrm{R}}^{d}$ in accordance to typical values of energy consumed by a UAV [37]. Moreover, we consider a fixed amount of energy $E_{\mathrm{F}}^{s}$ consumed by the site, which we expect to be higher than $E_{\mathrm{R}}^{d}[6]$. Focusing then on the battery levels $B_{\mathrm{MAX}}$ and $B_{\mathrm{MIN}}$, we set them in accordance to [8]. In addition, the amount of energy produced by one SP is retrieved from the data available for the location [38], by considering the month of June for the two scenarios.

In the next part, we consider the installation costs of the equipment, by adopting a setting similar to the one reported in [6]. Despite the site installation cost $C^{s}$ is much higher compared to the costs for buying a single UAV/SP/battery, we stress the fact that a single site may serve multiple areas, and hence the number UAVs/SPs/batteries installed on it may result in overall costs of these terms comparable to $C^{s}$.

Finally, the last rows of Tab. I report the setting for the maximum number of SPs $N_{\mathrm{SP}}^{\mathrm{MAX}}$ and the maximum number of batteries $N_{\mathrm{B}}^{\mathrm{MAX}}$. We set both of them to sufficiently large numbers, in order to guarantee that OPTRURALDESIGN and DIARIZE are able to return feasible solutions.

\section{RESULTS}

We code the OPTRURALDESIGN problem within the ILOG CPLEX Optimization Studio software (v.12.7.1) and run it on an a high performance computing cluster, composed of four nodes, each of them equipped with 32 cores and 64 GB of RAM, for a total computing power of around 1.5 TeraFlops/s. On the other hand, the DIARIZE algorithm is coded in Matlab, and run on a personal computer equipped with an Intel i5 processor at $2.7 \mathrm{GHz}$ and $8 \mathrm{~GB}$ of RAM.

\footnotetext{
${ }^{4}$ The investigation of more complex models to compute $C_{\mathrm{F}}^{s, s^{\prime}}$ is left for future work.
}

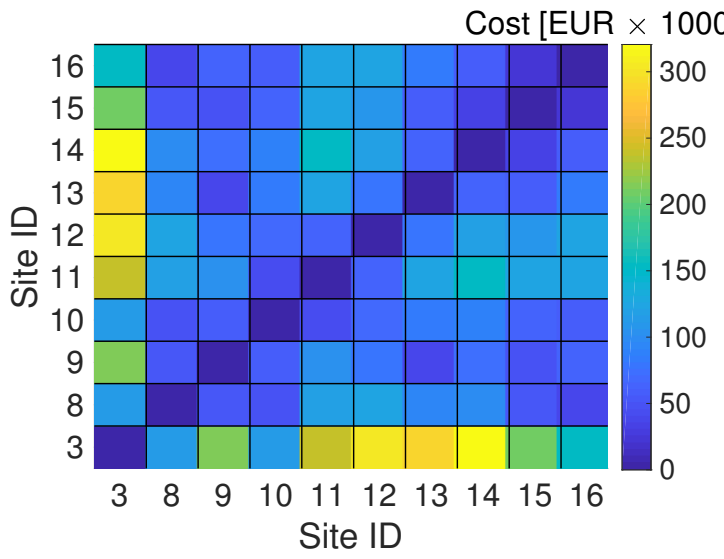

(a) Frascati Small

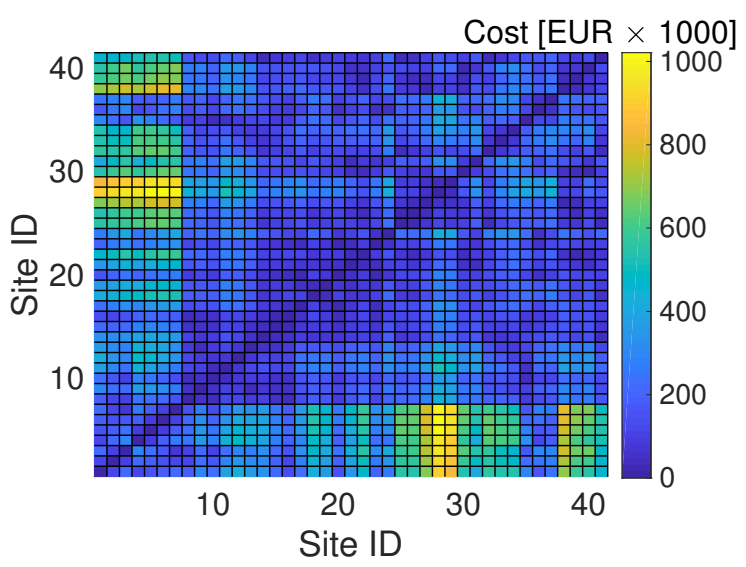

(b) Frascati Big

Fig. 3: Links installation costs $C_{\mathrm{F}}^{s, s^{\prime}}$ among all the possible site pairs.

Apart from OPTRURALDESIGN and DIARIZE, we also consider a classical approach, called REFDESIGN, as a term of comparison. ${ }^{5}$ In REFDESIGN, the coverage is provided by fixed BSs, mounted at the centers of the areas, and connected by means of a minimum cost fiber ring. Clearly, REFDESIGN does not exploit any UAV. In addition, we neglect the installation costs of SPs and batteries for this solution, in order to introduce a very optimistic assumption for this strategy.

For the performance assessment, we divide the experiments in the following sets: comparison of the algorithms, sensitivity analysis of the DIARIZE parameters, and results from large scenarios.

\section{A. Algorithms Comparison}

We solve OPTRURALDESIGN, DIARIZE and REFDESIGN over the Frascati Small scenario. We initially set $N_{\mathrm{MIN}}^{\mathrm{S}}=3$,

\footnotetext{
${ }^{5}$ At the time of writing this paper, OPTRURALDESIGN and DIARIZE are the only solutions jointly targeting: i) the selection of the installed ground sites, ii) the design of the optical fiber rings interconnecting the sites, ii) the dimensioning of each site in terms of SPs, batteries and used UAVs. Thus, there is not (yet) an existing best benchmark targeting a similar goal. Therefore, we select a classical approach based on fixed BSs as a term of comparison
} 
TABLE III: Algorithms comparison - Frascati Small scenario.

\begin{tabular}{|c|c|c|c|c|c|c|c|}
\hline & & \multicolumn{5}{|c|}{ Equipment Types } & Total Costs \\
\hline Cost $[€ \times 1000]$ & $\begin{array}{c}\text { Eq. } \\
\text { REFDESIGN } \\
\text { OPTRURALDESIGN } \\
\text { DIARIZE }\end{array}$ & $\begin{array}{c}\sum_{s} C^{s} y^{s} \\
400 \\
120 \\
120 \\
\end{array}$ & $\begin{array}{c}\sum_{s, s^{\prime}} C_{\mathrm{F}}^{s, s^{\prime}} f^{s, s^{\prime}} \\
300.01 \\
167.02 \\
167.02\end{array}$ & $\begin{array}{c}\sum_{s} C_{\mathrm{B}} N_{\mathrm{B}}^{s} \\
- \\
7.65 \\
7.65\end{array}$ & $\begin{array}{c}\sum_{s} C_{\mathrm{SP}} N_{\mathrm{SP}}^{s} \\
- \\
20 \\
23.2\end{array}$ & $\begin{array}{c}C_{\mathrm{D}} N_{\mathrm{D}} \\
- \\
86 \\
86 \\
\end{array}$ & $\begin{array}{l}\text { Eq. (20) } \\
700.01 \\
400.67 \\
403.87\end{array}$ \\
\hline Number & $\begin{array}{c}\text { Eq. } \\
\text { REFDESIGN } \\
\text { OPTRURALDESIGN } \\
\text { DIARIZE }\end{array}$ & $\begin{array}{c}\sum_{s} y^{s} \\
10 \\
3 \\
3\end{array}$ & $\begin{array}{c}\sum_{s, s^{\prime}} f^{s, s^{\prime}} \\
10 \\
3 \\
3\end{array}$ & $\begin{array}{c}\sum_{s} N_{\mathrm{B}}^{s} \\
- \\
51 \\
51\end{array}$ & $\begin{array}{c}\sum_{s} N_{\mathrm{SP}}^{s} \\
- \\
25 \\
29\end{array}$ & $\begin{array}{c}N_{\mathrm{D}} \\
- \\
20 \\
20\end{array}$ & $\begin{array}{l}20 \\
102 \\
106\end{array}$ \\
\hline
\end{tabular}

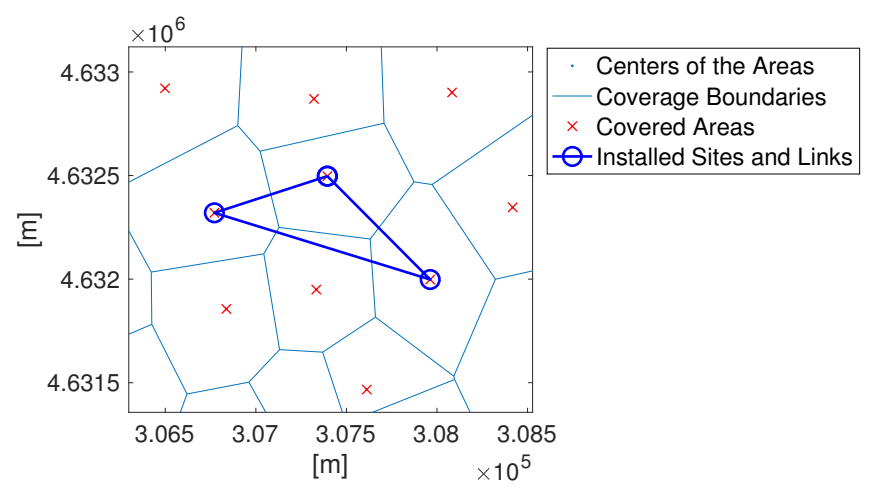

Fig. 4: Installed sites and fiber links (OPTRURALDESIGN/DIARIZE) - Frascati Small scenario.

$N_{\mathrm{MAX}}^{\mathrm{S}}=8$ and $I=20$ for DIARIZE. Moreover, we set a maximum time limit of 5 [h] for OptRURALDESIGN. Tab. III reports the breakdown of the results in terms of costs and number of installed devices for each solution, as well as the total numbers. Focusing first on the comparison between OptRURALDESIGN and REFDESIGN, the former is able to notably reduce the installation costs of sites and fibers compared to the latter, thanks to the exploitation of the UAVs. In particular, OPTRURALDESIGN requires the installation of 3 sites and the deployment of a ring with 3 links. On the other hand, RefDesign imposes to install 10 sites and 10 fiber links. In addition, the costs due to the installation of SPs and batteries are kept limited by OPTRURALDESIGN. Although a cost of $86[€ \times 1000]$ is experienced by installing the UAVs (and this cost is comparable to the one spent for the installation of a single site), the overall cost of OPTRURALDESIGN is much lower compared to REFDESIGN, with a cost saving of more than $42 \%$. Focusing then on the comparison between OPTRURALDESIGN and DIARIZE, we can note that both of them select the installation of the same sites and the same fiber links, as shown in Fig.4. Moreover, the total costs of batteries is the same for both solutions. At last, there is only a minor difference in terms of installed SPs, which is slightly higher in DIARIZE. Eventually, the total costs of OPTRURALDESIGN and DIARIZE are very close, with a relative difference of less than $0.8 \%$.

In the following, we compare in more detail the solutions retrieved by OptRURALDESIGN and DIARIZE. Fig. 4 reports the installed sites and the covered areas. Both the two solutions select the same site locations and the same fiber links. The sites that are installed are selected from the candidate sites located
TABLE IV: Breakdown of the installed SPs and batteries Frascati Small scenario.

\begin{tabular}{|c|c|c|c|c|}
\hline \multirow{2}{*}{ Metric } & \multirow{2}{*}{ Algorithm } & \multicolumn{3}{|c|}{ Site ID } \\
& & $\mathbf{9}$ & $\mathbf{1 0}$ & $\mathbf{1 5}$ \\
\hline \multirow{2}{*}{ Batteries } & OPTRURALDESIGN & 21 & 15 & 15 \\
\cline { 3 - 5 } & DIARIZE & 15 & 18 & 18 \\
\hline \multirow{2}{*}{ SPs } & OPTRURALDESIGN & 10 & 8 & 7 \\
\cline { 3 - 5 } & DIARIZE & 11 & 9 & 9 \\
\hline
\end{tabular}

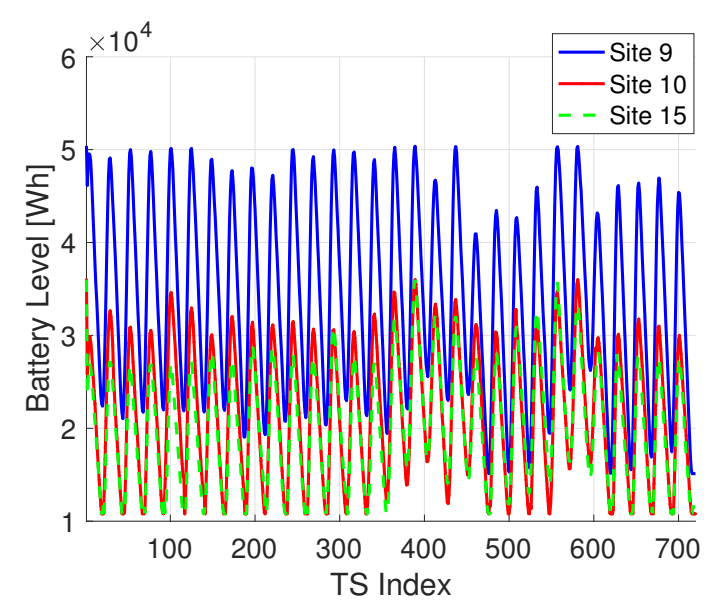

Fig. 5: Battery levels $B^{s}(t)$ vs. time - OptRuRALDESIGN, Frascati Small scenario.

in the central zone, where it is feasible to reach the areas that need to be covered. Tab. IV reports then the breakdown of the SPs and batteries installed in each site. Although the total number of installed batteries is the same in the two solutions (see Tab. III), their actual distribution over the sites is different. In addition, OPTRURALDESIGN requires less SPs compared to DIARIZE, and place them in a slight different way. This is due to the fact that the optimal solution is able to tackle the problem as a whole, while the heuristic works in a step-bystep manner. In particular, the DIARIZE algorithm computes the association of the areas to the sites in the first step, and this association is kept fixed for the whole set of TSs. On the other hand, OPTRURALDESIGN is able to change the area-tosite association on a TS-level, thus exploiting in a wiser way the energy available from the SPs. However, computing the optimal solution has a non-negligible cost. In our experiment, the time limit of $5[\mathrm{~h}]$ allowed retrieving a solution with less than $0.2 \%$ gap from the best integer one. On the contrary, DIARIZE requires less than 90 [s] to retrieve the solution.

Up to this point, a natural question is then: what is the trend 
TABLE V: Impact of the $I$ parameter for DIARIZE - Frascati Small scenario.

\begin{tabular}{|c|c|c|}
\hline Parameter & Comp. Time & Best Feasible Solution \\
\hline$I=5$ & $8.25[\mathrm{~s}]$ & $504.76[€ \times 1000]$ \\
\hline$I=20$ & $15.93[\mathrm{~s}]$ & $403.87[€ \times 1000]$ \\
\hline$I=40$ & $27.93[\mathrm{~s}]$ & $403.87[€ \times 1000]$ \\
\hline
\end{tabular}

TABLE VI: Impact of the $N_{\text {MAX }}^{\mathrm{S}}$ parameter for DIARIZE Frascati Small scenario.

\begin{tabular}{|l|c|c|}
\hline Parameter & Comp. Time & Best Feasible Solution \\
\hline$N_{\mathrm{MAX}}^{\mathrm{S}}=4$ & $12.31[\mathrm{~s}]$ & $403.87[€ \times 1000]$ \\
\hline$N_{\mathrm{MAX}}^{\mathrm{S}}=5$ & $19.48[\mathrm{~s}]$ & $403.87[€ \times 1000]$ \\
\hline$N_{\mathrm{MAX}}^{\mathrm{S}}=6$ & $13.29[\mathrm{~s}]$ & $412.79[€ \times 1000]$ \\
\hline$N_{\mathrm{MAX}}^{\mathrm{S}}=7$ & $18.18[\mathrm{~s}]$ & $403.87[€ \times 1000]$ \\
\hline$N_{\mathrm{MAX}}^{\mathrm{S}}=8$ & $15.93[\mathrm{~s}]$ & $403.87[€ \times 1000]$ \\
\hline
\end{tabular}

of the battery levels $B^{s}(t)$ in the installed sites? To answer this question, Fig. 5 reports $B^{s}(t)$ for the three sites selected by OPTRURALDESIGN. Interestingly, the battery level is not constant. During the night, it tends to be decreased, due the fact that: i) the energy from the SPs is not available, and ii) a non-negligible amount of energy is consumed by the site and the UAV(s) recharging on it. On the other hand, the battery level is increased during the day, thanks to the energy produced by SPs. Clearly, since site 9 has more batteries compared to the other sites, its battery levels are in general higher than the ones of site 10 and site 15 .

\section{B. Sensitivity Analysis of the DIARIZE parameters}

We analyze the impact of varying the input parameters of DIARIZE on the Frascati Small scenario. We initially consider the variation of $I=\{5,20,40\}$, while we keep $N_{\mathrm{MAX}}^{\mathrm{S}}=8$. We recall that $I$ is used to control the number of cycles of the k-medoids routine performed in line 1 of Alg. 2. Moreover, we point out that $I$ governs also the cycles of the loop appearing in line 4 of Alg. 2. Tab. V reports the obtained results in terms of total cost of the best feasible solution and total computation time. As expected, a lower $I$ results in a shorter computation time, thanks to the fact that DIARIZE reduces the required number of cycles. However, the quality of the solution may be impacted. For example, when $I=\{5\}$ the total costs are increased by more than $24 \%$ compared to the $I=\{20\}$ case. On the other hand, a setting of $I=\{20\}$ already ensures a good quality in the obtained solution.

In the following, we vary $N_{\mathrm{MAX}}^{\mathrm{S}}=\{4,5,6,7,8\}$, while we keep a $I=20$. We recall that the $N_{\mathrm{MAX}}^{\mathrm{S}}$ parameter is used to select the maximum number of installed sites, which is exploited by the k-medoids routine in line 1 of Alg. 2 and by the for cycle in line 3. Tab. VI reports the obtained results. Clearly, since in this case there are only 3 sites installed in the best solution, it is already possible to retrieve a good solution even when $N_{\mathrm{MAX}}^{\mathrm{S}}=4$. On the other hand, minor variations in the computation times and in the total costs are experienced for the other values of $N_{\mathrm{MAX}}^{\mathrm{S}}$.

In the next part, we tried to answer to the question: How to set $N_{\text {MIN }}^{\mathrm{S}}$ without any a-priori information from the scenario? To answer this question, Fig. 6 reports the transient behavior of DIARIZE, obtained by placing a probe at line 15 of Alg. 2,

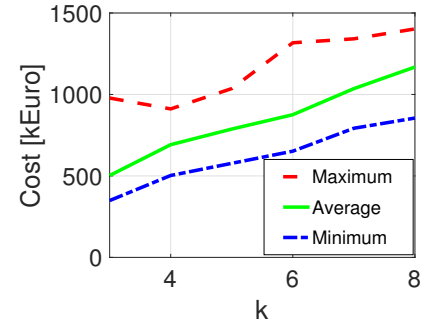

(a) Cost variation

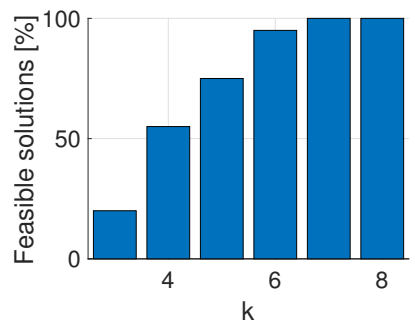

(b) Percentage of feasible solutions
Fig. 6: DIARIZE transient behavior vs. $k$ - Frascati Small scenario.

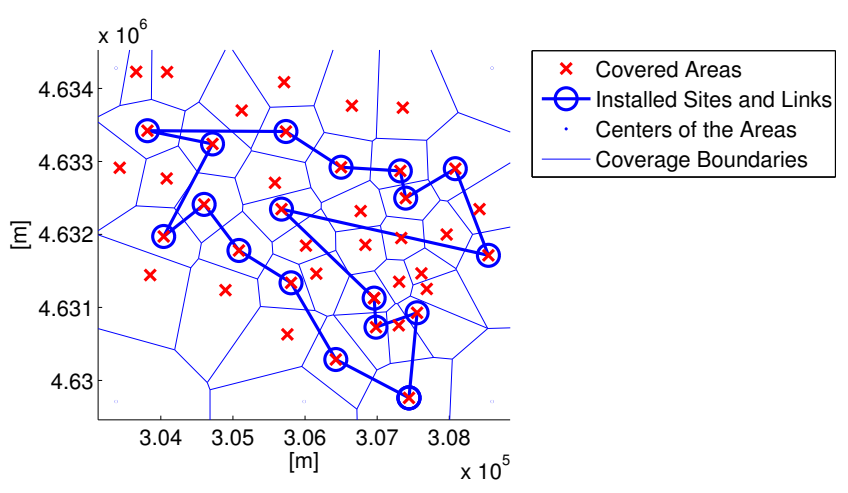

Fig. 7: Installed sites and fiber links - DIARIZE algorithm, Frascati Big scenario.

and by collecting the total cost and the feasibility for each solution indexed by $k .^{6}$ Fig. 6a reports minimum, average and total costs for the solutions found vs. $k$. Clearly, the cost tends to increase with $k$, which is the number of installed sites in the current iteration. Fig. $6 \mathrm{~b}$ then reports the percentage of feasible solutions over the total ones. When $k$ is low, few feasible solutions can be found (i.e., around $20 \%$ when $k=3$ ). On the contrary, when $k$ is increased, the percentage of feasible solutions is also increased. However, this results also in an increase of the total costs. The two metrics could be used to tune the $N_{\mathrm{MIN}}^{\mathrm{S}}$ parameter: in a general scenario, it may happen that, for a specific value of $N_{\text {MIN }}^{\mathrm{S}}$, no feasible solutions can be found. Therefore, $N_{\text {MIN }}^{\mathrm{S}}$ should be increased until a feasible solution becomes available. However, it is also important to consider the increase in the total costs.

\section{Results from Large Scenarios}

We consider the Frascati Big scenario to run the REFDESIGN and DIARIZE algorithms. Focusing on DIARIZE, we set $N_{\mathrm{MIN}}^{\mathrm{S}}=10, N_{\mathrm{MAX}}^{\mathrm{S}}=30, I=40$. In this case, solving the problem with OPTRURALDESIGN is not practically feasible, due to the very large problem size, coupled with its proven complexity. On the other hand, DIARIZE is able to retrieve a solution in less than 5 [minutes]. Tab. VII compares the results obtained from DIARIZE and REFDESIGN. Interestingly, DIARIZE is able to notably reduce the site installation

\footnotetext{
${ }^{6}$ During this experiment we set $N_{\mathrm{MIN}}^{\mathrm{S}}=3, N_{\mathrm{MAX}}^{\mathrm{S}}=8, I=40$.
} 
TABLE VII: Algorithms comparison - Frascati Big scenario.

\begin{tabular}{|c|c|c|c|c|c|c|c|}
\hline & & \multirow{2}{*}{\multicolumn{5}{|c|}{$\begin{array}{r}\text { Equipment Types } \\
\text {. }\end{array}$}} & \multirow{2}{*}{ Total Costs } \\
\hline & & & & & & UAVs & \\
\hline Cost $[€ \times 1000]$ & $\begin{array}{c}\text { Eq. } \\
\text { REFDESIGN } \\
\text { DIARIZE }\end{array}$ & $\begin{array}{c}\sum_{s} C^{s} y^{s} \\
1640 \\
720\end{array}$ & $\begin{array}{c}\sum_{s, s^{\prime}} C_{\mathrm{F}}^{s, s^{\prime}} f^{s, s^{\prime}} \\
3169 \\
1849.75\end{array}$ & $\begin{array}{c}\sum_{s} C_{\mathrm{B}} N_{\mathrm{B}}^{s} \\
- \\
40.05\end{array}$ & $\begin{array}{c}\sum_{s} C_{\mathrm{SP}} N_{\mathrm{SP}}^{s} \\
-- \\
121.6\end{array}$ & $\begin{array}{c}C_{\mathrm{D}} N_{\mathrm{D}} \\
- \\
352.6\end{array}$ & $\begin{array}{c}\text { Eq. (20) } \\
4809 \\
3084\end{array}$ \\
\hline Number & $\begin{array}{c}\text { Eq. } \\
\text { REFDESIGN } \\
\text { DIARIZE }\end{array}$ & $\begin{array}{c}\sum_{s} y^{s} \\
41 \\
18\end{array}$ & $\begin{array}{c}\sum_{s, s^{\prime}} f^{s, s^{\prime}} \\
18\end{array}$ & $\begin{array}{c}\sum_{s} N_{\mathrm{B}}^{s} \\
- \\
267\end{array}$ & $\begin{array}{c}\sum_{s} N_{\mathrm{SP}}^{s} \\
- \\
152\end{array}$ & $N_{\mathrm{D}}$ & $\begin{array}{c}82 \\
535\end{array}$ \\
\hline
\end{tabular}

TABLE VIII: Breakdown of the elements installed by DIARIZE - Frascati Big scenario.

\begin{tabular}{|c|c|c|c|c|}
\hline Site ID & Batteries & SPs & Served Areas & UAVs \\
\hline $4,12,36,37$ & 12 & 7 & 1 & 2 \\
$13,19,14,21,22,26,30,41$ & 15 & 8 & 2 & 4 \\
$1,9,35$ & 18 & 9 & 3 & 6 \\
$6,23,27$ & 15 & 11 & 4 & 8 \\
\hline
\end{tabular}

costs, as well as the fiber costs. Overall, the total cost reduction achieved by DIARIZE is more than $35 \%$ compared to REFDESIGN. This result is achieved by installing only 18 sites over 41 candidate ones, and by placing the optical ring in such a way that reduces the fiber cost, as shown in Fig. 7.

In the following, we provide more details about the performance results of the DIARIZE algorithm. Tab. VIII reports the installed sites and their configuration in terms of: number of batteries, number of SPs, number of served areas and number of used UAVs. Interestingly, there is not a unique configuration that is applied to all the installed sites. On the contrary, four distinct configurations are used, each of them characterized by a set of SPs, batteries, UAVs and number of areas that are covered. This outcome further motivates our approach, which is based on the idea of finding the optimal configuration for each installed site, in order to minimize the total installation costs. In addition, we can note that the higher is the number of used UAVs, the higher is in general the number of installed SPs and batteries. This is an expected result, since the larger is the set of UAVs performing recharging actions, the higher will be their total energy demand. However, we can note that the increase in the number of SPs is not always followed by an increase in the number of batteries. This trend appears e.g., in the last two configurations reported in the bottom of the table. Therefore, a combined approach, like the one pursued in this paper, is essential to: i) minimize the total costs and ii) find the right combination of elements that need to be installed in order to satisfy the constraints.

In the following, we provide additional details on the performance of the DIARIZE, by analyzing the impact of the variation of the number of cycles $I$. Tab. IX reports the obtained results. By increasing $I$, it is possible to reduce the total costs, due to the fact that the quality of the best solution is improved (a similar trend has been observed also in the Frascati Small scenario). However, the increase of $I$ has also an impact on the computation time, which tends to be increased too. Interestingly, DIARIZE is always very effective in limiting the total computation time, which is always less than 5 minutes, even when $I=40$.
TABLE IX: Impact of the $I$ parameter for DIARIZE - Frascati Big scenario.

\begin{tabular}{|c|c|c|}
\hline Parameter & Comp. Time & Best Feasible Solution \\
\hline$I=5$ & $37.44[\mathrm{~s}]$ & $3584[€ \times 1000]$ \\
\hline$I=20$ & $149.77[\mathrm{~s}]$ & $3521[€ \times 1000]$ \\
\hline$I=40$ & $299.53[\mathrm{~s}]$ & $3084[€ \times 1000]$ \\
\hline
\end{tabular}

\section{DISCUSSION}

In this section, we provide a brief discussion of our findings. First of all, our work includes the REFDESIGN solution, in which fixed BSs are used, as a term of comparison. The shortcomings of the REFDESIGN scheme are larger installation costs that are paid compared to OptRURALDESIGN and DIARIZE, since more fibers and/or physical infrastructure have to be installed over the territory. A natural question can be then: what are the shortcomings of the REFDESIGN scheme in terms of coverage compared to OPTRURALDESIGN and DIARIZE? To this aim, we point out that the set of areas are given as input to our problems. More in depth, the size of each area is already shaped to guarantee an adequate level of coverage when it is served either by a fixed BS or a UAV. In our work, in fact, we have assumed area sizes in the order of square kilometers at most, a value completely in line with coverage capabilities of currently deployed mobile networks [6] and UAV-based ones [32]. Therefore, when an area is served by either a fixed BS with REFDESIGN or a UAV with OPTRURALDESIGN and DIARIZE, it is fully covered. This alleviated us from the need of including a detailed mathematical model to ensure to coverage, which would dramatically increase the complexity of the considered problem, due to the fact that the coverage has to be evaluated over each Test Point (TP) in each area [40]. Eventually, we point out that the coverage evaluation is important in scenarios affected by strong sources of interference (like for example urban deployments, see e.g., [41]) and/or when the coverage radius of each BS is very large [6]. On the other hand, our scenarios are based on rural areas of moderate size, in which the negative effects of interference and/or large distance from the serving BS are mitigated. The evaluation of detailed coverage models is therefore left for future work.

Our work is tailored to the minimization of the CAPital EXpenditures (CAPEX) costs of an architecture composed of ground sites, solar panels, batteries, fibers and UAVs. Specifically, each UAV is assumed to stay in the air for one TS, whose duration is set to one hour in our scenarios, in order to have sufficient time to complete the coverage mission. The duration of the UAV mission is line with currently available UAVs (see e.g., [39]). Once the mission is completed, the UAV 
comes back to a ground site to recharge itself. We assume that also the recharge takes one time slot, which is again a realistic parameter for currently available UAVs (see e.g., [39]). Given this picture, a natural question can be: why are the OPerating EXpenditures (OPEX) costs not considered? In other works, like for example [6], the OPEX costs of a UAV-based architecture are expressed in terms of: i) energy required to the grid and ii) maintenance costs for ground sites. Differently from [6], in this work we consider a completely self-sustainable architecture, in which the energy is solely provided by solar panels and batteries. Therefore, no energy is required to the grid. As a consequence, the energy required to recharge the UAVs does not impact the OPEX. Focusing then on the maintenance costs, in [6] they are proportional to the number of installed ground sites. Since a UAV-based architeture requires less ground sites compared to a reference architecture composed of fixed BSs, we can conclude that the OPEX of OPTRURALDESIGN and DIARIZE is lower compared to the one of REFDESIGN.

In addition, we stress the fact that, in our work, the QoS is expressed as a continuous coverage over a set of areas. This constraint is ensured by all the algorithms taken under consideration. Therefore, we do the comparison of REFDESIGN, DIARIZE and OPTRURALDESIGN under the same area QoS. Clearly, the evaluation of the QoS could be done also to single users, that is: given a UAV that is covering an area, how to wisely manage the radio resources of the UAV among the users in the area, in order to maximize their QoS? This management problem is also interesting, although complementary to the dimensioning problem faced by DIARIZE and OPTRURALDESIGN. Actually, the output of our approach (in terms e.g., of installed elements) can be used as input to the management problem, in which the QoS of the users is optimized, through the management of the radio resources provided by the UAVs.

Eventually, another aspect related to QoS is the amount of capacity that each site has to reserve to each connected area. With the REFDESIGN approach, each site serves a single area, and therefore each area receives the maximum value of capacity. On the other hand, for both DIARIZE and OPTRURALDESIGN, a single site may serve multiple areas, due to the fact that different UAVs, each of them serving an area, are connected to the same site. In order to evaluate this aspect, which may impact the performance to users, let us denote with $T^{s}$ the total capacity that is available at site $s$ to collect the traffic coming from the served area(s). Let us denote with $R_{a}^{s}$ the amount of capacity that area $a$ receives from site $s$, thanks to the fact that there is a UAV serving $a$, and this UAV is connected to $s$. In the Frascati Small scenario, both DIARIZE and OPTRURALDESIGN install the same 3 sites over the territory. By inspecting the $x_{d, a}^{s}(t)$ variables, we obtain that 4 areas are served by UAVs connected to Site 9, 3 areas are served by UAVs connected to Site 10 and the remaining 3 areas are served by UAVs connected to Site 15 . By assuming for simplicity $T^{s}=1$ [unit] $\forall s \in \mathcal{S}$ and a proportional splitting of the site capacity assigned to each area, we obtain the values of $R_{a}^{s}$ reported in Tab. X.

Interestingly, each area receives on average an amount of
TABLE X: Site capacity $R_{a}^{s}$ of DIARIZE / OPTRURALDESIGN - Frascati Small Scenario.

\begin{tabular}{|c|c|c|c|}
\hline \multirow{2}{*}{ Area ID } & \multicolumn{3}{|c|}{ Site ID } \\
& 9 & 10 & 15 \\
\hline 3 & - & $1 / 3$ & - \\
8 & $1 / 4$ & - & - \\
9 & $1 / 4$ & - & - \\
10 & - & $1 / 3$ & - \\
11 & - & $1 / 3$ & - \\
12 & $1 / 4$ & - & - \\
13 & $1 / 4$ & - & - \\
14 & - & - & $1 / 3$ \\
15 & - & - & $1 / 3$ \\
16 & - & - & $1 / 3$ \\
\hline
\end{tabular}

site capacity reduced between $1 / 3$ and $1 / 4$ compared to the maximum value $T^{s}$. On the other hand, the REFDESIGN approach always assumes the installation of a site in each area, and therefore $R_{a}^{s}=T^{s}=1 \quad \forall a \in \mathcal{A}, s \in \mathcal{S}$ for this strategy. Now, a natural question is then: how to compare the different approaches considering $R_{a}^{s}$ ? A first observation is that, for a given scenario solved by DIARIZE/OPTRURALDESIGN, the less sites are installed compared to REFDESIGN, the lower is the value of $R_{a}^{s}$. However, this parameter can be easily controlled both inside the model and the algorithm, by imposing a constraint on the maximum number of areas that can be served by each site in each TS. More formally, we introduce the following constraint:

$$
\sum_{a \in \mathcal{A}} \sum_{d \in \mathcal{D}} x_{d, a}^{s}(t) \leq O^{\mathrm{MAX}} \quad \forall s \in \mathcal{S}, t \in \mathcal{T}
$$

where $O^{\mathrm{MAX}}$ is the maximum number of areas that can be served by the UAVs connected to the same site. In order to compare the DIARIZE/OPTRURALDESIGN and the REFDESIGN approaches under the same capacity assigned to each area, we set $O^{\mathrm{MAX}}=1$. Moreover, we remind that, with REFDESIGN, we adopt the assumption that each site is connected to the grid, and therefore no SPs/batteries have to be installed. In order to make a fair comparison, we introduce this assumption also for our algorithms. We then run OPTRURALDESIGN with $O^{\mathrm{MAX}}=1$, and we get the results reported in Tab.XI. By analyzing the table, we can conclude that, when each site has to guarantee the maximum capacity to each area, the UAV-based solution requires higher costs compared to the reference approach. This is due to the following reasons: i) the number of sites installed by OPTRURALDESIGN is equal to the number of areas, ii) additional costs are paid by OPTRURALDESIGN compared to REFDESIGN, due to the exploitation of the UAVs. However, we point out that: i) the setting of $O^{\mathrm{MAX}}=1$ is an extreme case, ii) users in the rural zones are more willing to receive a mobile connectivity, rather than having the same capacity offered in urban zones [6], iii) the operator will not likely deploy a site in each area, due to the large installation costs that are incurred, iv) the capacity of the UAV-based solutions could be improved by allowing multiple UAVs (connected to different sites) serving the same area at the same time. However, we also point out that a detailed investigation of such aspects is left for future work. 
TABLE XI: Algorithms comparison with $O^{\mathrm{MAX}}=1$ - Frascati Small scenario.

\begin{tabular}{|c|c|c|c|c|c|c|c|}
\hline & & & $\begin{aligned} \text { Equ } & \end{aligned}$ & ment Types & & & Total Costs \\
\hline Cost $[€ \times 1000]$ & $\begin{array}{c}\text { Eq. } \\
\text { REFDESIGN } \\
\text { OPTRURALDESIGN }\end{array}$ & $\begin{array}{c}\sum_{s} C^{s} y^{s} \\
400 \\
400\end{array}$ & $\begin{array}{c}\sum_{s, s^{\prime}} C_{\mathrm{F}}^{s, s^{\prime}} f^{s, s^{\prime}} \\
300.01 \\
300.01\end{array}$ & $\begin{array}{c}\sum_{s} C_{\mathrm{B}} N_{\mathrm{B}}^{s} \\
- \\
-\end{array}$ & $\begin{array}{c}\sum_{s} C_{\mathrm{SP}} N_{\mathrm{SP}}^{s} \\
- \\
-\end{array}$ & $\begin{array}{c}C_{\mathrm{D}} N_{\mathrm{D}} \\
- \\
86\end{array}$ & $\begin{array}{c}\text { Eq. (20) } \\
700.01 \\
786.01\end{array}$ \\
\hline Number & $\begin{array}{c}\text { Eq. } \\
\text { REFDESIGN } \\
\text { OPTRURALDESIGN }\end{array}$ & $\begin{array}{c}\sum_{s} y^{s} \\
10 \\
10\end{array}$ & $\begin{array}{c}\sum_{s, s^{\prime}} f^{s, s^{\prime}} \\
10 \\
10\end{array}$ & $\begin{array}{c}\sum_{s} N_{\mathrm{B}}^{s} \\
-\end{array}$ & $\begin{array}{c}\sum_{s} N_{\mathrm{SP}}^{s} \\
-\end{array}$ & $\begin{array}{c}N_{\mathrm{D}} \\
- \\
20\end{array}$ & $\begin{array}{l}- \\
20 \\
40\end{array}$ \\
\hline
\end{tabular}

\section{CONCLUSiOnS AND Future WORK}

We have targeted the problem of minimizing the installation costs for a UAV-based cellular architecture tailored to serve rural areas. We have initially formulated the innovative OPTRURALPLAN problem, which is able to select the sites that need to be installed, the set of optical fiber links that interconnect the sites through a ring, as well the dimensioning of each site in terms of number of SPs and number of batteries. The presented formulation takes into account the UAVs covering constraints, the recharging actions of the UAVs over time, as well as the constraints relative to installation of sites, SPs and batteries. In the following, we have designed the DIARIZE algorithm, which is able to retrieve a solution in a reasonably low amount of time. The results obtained over the Frascati Small scenario show that DIARIZE performs close to OPTRURALPLAN in terms of total installation costs. In addition, the analysis on the Frascati Big scenario reveals that DIARIZE is able to notably reduce the costs compared to a classical REFDESIGN algorithm, by applying different configurations of UAVs, SPs and batteries to the installed sites.

As next step, we plan a variety of research activities. First, the evaluation of longer missions performed by the UAVs may be an interesting option. Second, we plan to study the impact of the uncertainty in the SP energy (e.g., due to the presence of bad weather conditions). Third, we will introduce the evaluation of the Quality of Service (QoS) perceived by users and the level of coverage. In this case, the integration of our approach with solutions providing the placement of the UAVs in the 3D space may be a promising goal.

\section{ACKNOWLEDGEMENTS}

This work has been partially supported by the University of Rome Tor Vergata BRIGHT project (Mission Sustainability Call).

\section{REFERENCES}

[1] L. Chiaraviglio, L. Amorosi, N. Blefari-Melazzi, P. Dell'Olmo, P. Monti, and C. Natalino, "Optimal design of 5G networks in rural zones with UAVs, optical rings, solar panels and batteries," in International Conference on Transparent Optical Networks (ICTON), Bucharest, Romania, pp. 1-4, July 2018.

[2] "International Telecommunication Union (ITU), ICT Facts and Figures the world in 2015." http://www.itu.int/en/ITU-D/Statistics/Pages/facts/, last accessed in July 2018.

[3] "World internet penetration rates." http://www.internetworldstats.com/ stats.htm, last accessed in July 2018.

[4] M. Eriksson and J. Van de Beek, "Is Anyone Out There? 5G, Rural Coverage, and the Next One Billion." https://www.comsoc.org/ctn/ anyone-out-there-5g-rural-coverage-and-next-1-billion, last accessed in July 2018., 2015.
[5] J. G. Andrews, S. Buzzi, W. Choi, S. V. Hanly, A. Lozano, A. C. Soong, and J. C. Zhang, "What will 5G be?," IEEE Journal on Selected Areas in Communications, vol. 32, no. 6, pp. 1065-1082, 2014.

[6] L. Chiaraviglio, N. Blefari-Melazzi, W. Liu, J. A. Gutierrez, J. van de Beek, R. Birke, L. Chen, F. Idzikowski, D. Kilper, P. Monti, A. Bagula, and J. Wu, "Bringing 5G into Rural and Low-Income Areas: Is it Feasible?," IEEE Communications Standards Magazine, vol. 1, no. 3, pp. 50-57, 2017.

[7] J. Wu, S. Guo, H. Huang, W. Liu, and Y. Xiang, "Information and Communications Technologies for Sustainable Development Goals: State-ofthe-Art, Needs and Perspectives," IEEE Communications Surveys and Tutorials, vol. on press, 2018.

[8] Y. Zhang, M. Meo, R. Gerboni, and M. A. Marsan, "Minimum cost solar power systems for lte macro base stations," Computer Networks, vol. 112, pp. 12-23, 2017.

[9] M. Mozaffari, W. Saad, M. Bennis, and M. Debbah, "Unmanned aerial vehicle with underlaid device-to-device communications: Performance and tradeoffs," IEEE Transactions on Wireless Communications, vol. 15, no. 6, pp. 3949-3963, 2016.

[10] M. Mozaffari, W. Saad, M. Bennis, and M. Debbah, "Efficient deployment of multiple unmanned aerial vehicles for optimal wireless coverage.," IEEE Communications Letters, vol. 20, no. 8, pp. 16471650, 2016.

[11] M. Mozaffari, W. Saad, M. Bennis, and M. Debbah, "Drone small cells in the clouds: Design, deployment and performance analysis," in IEEE GLOBECOM, San Diego, CA, pp. 1-6, December 2015.

[12] M. Mozaffari, W. Saad, M. Bennis, and M. Debbah, "Optimal transport theory for power-efficient deployment of unmanned aerial vehicles," in IEEE ICC, Kuala Lumpur, Malaysia, pp. 1-6, May 2016.

[13] A. Merwaday and I. Guvenc, "UAV assisted heterogeneous networks for public safety communications," in IEEE WCNC Workshops, Instabul, Turkey, pp. 329-334, May 2015.

[14] M. Mozaffari, W. Saad, M. Bennis, and M. Debbah, "Optimal transport theory for cell association in uav-enabled cellular networks," IEEE Communications Letters, vol. 21, no. 9, pp. 2053-2056, 2017.

[15] Q. Wu, Y. Zeng, and R. Zhang, "Joint trajectory and communication design for multi-uav enabled wireless networks," IEEE Transactions on Wireless Communications, vol. 17, no. 3, pp. 2109-2121, 2018.

[16] E. Kalantari, M. Z. Shakir, H. Yanikomeroglu, and A. Yongacoglu, "Backhaul-aware robust 3d drone placement in $5 \mathrm{~g}+$ wireless networks," in IEEE ICC Workshops, Paris, France, pp. 109-114, May 2017.

[17] E. Kalantari, H. Yanikomeroglu, and A. Yongacoglu, "On the number and 3d placement of drone base stations in wireless cellular networks," in IEEE VTC-Fall, Montreal, Canada, pp. 1-6, September 2016.

[18] M. M. Azari, F. Rosas, K.-C. Chen, and S. Pollin, "Optimal UAV positioning for terrestrial-aerial communication in presence of fading," in IEEE GLOBECOM, Washington, DC USA, pp. 1-7, December 2016.

[19] J. Lyu, Y. Zeng, R. Zhang, and T. J. Lim, "Placement optimization of uav-mounted mobile base stations," IEEE Communications Letters, vol. 21, no. 3, pp. 604-607, 2017.

[20] R. Dai, S. Fotedar, M. Radmanesh, and M. Kumar, "Quality-aware uav coverage and path planning in geometrically complex environments," $\mathrm{Ad}$ Hoc Networks, vol. 73, pp. 95-105, 2018.

[21] G. Piro, M. Miozzo, G. Forte, N. Baldo, L. A. Grieco, G. Boggia, and P. Dini, "Hetnets powered by renewable energy sources: Sustainable next-generation cellular networks," IEEE Internet Computing, vol. 17, no. 1, pp. 32-39, 2013.

[22] V. Chamola and B. Sikdar, "Solar powered cellular base stations: current scenario, issues and proposed solutions," IEEE Communications magazine, vol. 54, no. 5, pp. 108-114, 2016.

[23] M. A. Marsan, G. Bucalo, A. Di Caro, M. Meo, and Y. Zhang, "Towards zero grid electricity networking: Powering bss with renewable energy sources," in IEEE ICC Workshops, Budapest, Hungary, pp. 596-601, June 2013. 
[24] A. P. Couto da Silva, D. Renga, M. Meo, and M. Ajmone Marsan, "The impact of quantization on the design of solar power systems for cellular base stations," IEEE Transaction on Green Communications and Networking, vol. 2, no. 1, pp. 260-274, 2018.

[25] O. Onireti, J. Qadir, M. A. Imran, and A. Sathiaseelan, "Will 5g see its blind side? evolving $5 \mathrm{~g}$ for universal internet access," in $A C M$ SIGCOMM Global Access to the Internet for All (GAIA) Workshop, Florianopolis, Brazil, pp. 1-6, August 2016.

[26] M. Fiorani, B. Skubic, J. Mårtensson, L. Valcarenghi, P. Castoldi, L. Wosinska, and P. Monti, "On the design of $5 \mathrm{~g}$ transport networks," Photonic network communications, vol. 30, no. 3, pp. 403-415, 2015.

[27] M. Fiorani, S. Tombaz, J. Mårtensson, B. Skubic, L. Wosinska, and P. Monti, "Modeling energy performance of c-ran with optical transport in 5g network scenarios," Journal of Optical Communications and Networking, vol. 8, no. 11, pp. B21-B34, 2016.

[28] E. Wong, E. Grigoreva, L. Wosinska, and C. M. Machuca, "Enhancing the survivability and power savings of $5 \mathrm{~g}$ transport networks based on dwdm rings," IEEE/OSA Journal of Optical Communications and Networking, vol. 9, no. 9, pp. D74-D85, 2017.

[29] Y. Zeng, R. Zhang, and T. J. Lim, "Wireless communications with unmanned aerial vehicles: opportunities and challenges," IEEE Communications Magazine, vol. 54, no. 5, pp. 36-42, 2016.

[30] M. Mozaffari, W. Saad, M. Bennis, Y.-H. Nam, and M. Debbah, "A tutorial on UAVs for wireless networks: Applications, challenges, and open problems," IEEE Commmunications Surveys \& Tutorials, 2019.

[31] S. Chandrasekharan, K. Gomez, A. Al-Hourani, S. Kandeepan, T. Rasheed, L. Goratti, L. Reynaud, D. Grace, I. Bucaille, T. Wirth, and S. Allsopp, "Designing and implementing future aerial communication networks," IEEE Communications Magazine, vol. 54, no. 5, pp. 26-34, 2016.

[32] A. Al-Hourani, S. Kandeepan, and S. Lardner, "Optimal lap altitude for maximum coverage," IEEE Wireless Communications Letters, vol. 3, no. 6, pp. 569-572, 2014.

[33] L. Chiaraviglio, N. Blefari-Melazzi, W. Liu, J. A. Gutierrez, J. van de Beek, R. Birke, L. Chen, F. Idzikowski, D. Kilper, P. Monti, and J. Wu, "5G in rural and low-income areas: Are we ready?" in Proc. of ITU Kaleidoscope: ICTs for a Sustainable World, Bangkok, Thailand, pp. 18, 2016.

[34] M. R. Garey and D. S. Johnson, Computers and Intractability: A Guide to the Theory of NP-Completeness. Freeman, 1979.

[35] A. Schrijver, Combinatorial optimization: polyhedra and efficiency, vol. 24. Springer Science \& Business Media, 2003.

[36] L. Kaufman and P. Rousseeuw, Clustering by means of medoids. NorthHolland, 1987.

[37] T. Dietrich, S. Krug, and A. Zimmermann, "An empirical study on generic multicopter energy consumption profiles," in IEEE International Systems Conference (SysCon), Montreal, Canada, pp. 1-6, April 2017.

[38] B. Marion and M. Anderberg, "PVWATTS - an online performance calculator for grid-connected PV systems," in Proceedings of the ASES Solar Conference, Madison, Wisconsin, pp. 119-124, June 2000.

[39] "US-1 Law Enforcement Drone Datasheet." https://impossible.aero/wp-content/uploads/2019/03/

US-1-Impossible-Drone-Sheet-Law-Enforcement-V2.pdf, last accessed in June 2019.

[40] E. Amaldi, A. Capone, and F. Malucelli, "Radio planning and coverage optimization of $3 \mathrm{~g}$ cellular networks," Wireless Networks, vol. 14, no. 4, pp. $435-447,2008$

[41] L. Chiaraviglio, J. Galán-Jiménez, M. Fiore, and N. Blefari-Melazzi, "Not in my neighborhood: A user equipment perspective of cellular planning under restrictive emf limits," IEEE Access, vol. 7, pp. 6161$6185,2018$. 


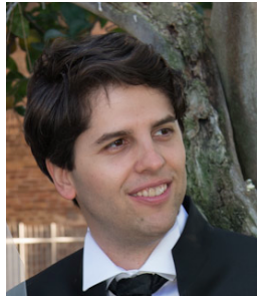

Luca Chiaraviglio is a Tenure Track Assistant Professor at the Networking Group in the Department of Electronic Engineering of University of Rome Tor Vergata. He holds a Ph.D. in Telecommunication and Electronics Engineering, obtained from Politecnico di Torino, Italy. He has spent research periods abroad in several international institutions, including: Boston University (USA), INRIA Sophia Antipolis (France), Auckland University of Technology (New Zealand) and ETECSA S.A. (Cuba).

Luca has co-authored $130+$ publications in international journals, books and conferences, and he has collaborated with more than 200+ co-authors. He participates in the TPC of top-leading conferences, including IEEE INFOCOM, IEEE GLOBECOM, IEEE ICC, IEEE VTC and IEEE GlobalSIP. Moreover, he is in the Editorial Board of IEEE Communications Magazine, IEEE Access and IEEE Transactions on Green Communications and Networking.

$\mathrm{He}$ is currently the coordinator of the national project BRIGHT: Bringing 5G Connectivity in Rural and Low-Income Areas. During the last years, he has been involved in different European projects, such as H2020 5G-EVE, H2020 Superfluidity, FP7 Trend, FP7 EcoNet, and FP7 Bone.

Luca has received the Best Paper Award in different conferences, including IEEE VTC and ICIN. Some of his papers are listed as Best Readings on Green Communications by IEEE. Moreover, he has been recognized as an author in the top $1 \%$ most highly cited papers in the ICT field worldwide. His papers "Optimal Energy Savings in Cellular Access Networks" and "Reducing Power Consumption in Backbone Networks" are the most cited papers from all IEEE ICC conferences and IEEE ICC workshops in the period 2009-2018 (Source: Scopus).

$\mathrm{He}$ also is an IEEE Senior Member and a founding member of the IEEE Communications Society Technical Subcommittee on Green Communications and Computing.

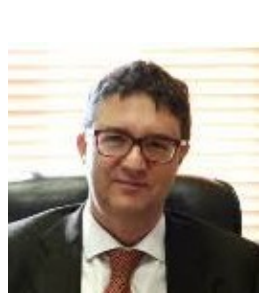

Nicola

Blefari-Melazzi (http://blefari.eln.uniroma2.it/) is a full Professor of Telecommunications at the University of Roma Tor Vergata, where he served as Chair of the $\mathrm{PhD}$ program in Telecommunications Engineering, Chair of the undergraduate and graduate programs in Telecommunications Engineering and Chair of the Department of Electronic Engineering. He is currently the Director of CNIT (National InterUniversity Consortium for Telecommunications, http://www.cnit.it/), a non-profit Consortium among 37 Italian Universities. More than 1,300 people, belonging to the participating universities, collaborate with CNIT, while the number of own-employees is more than 100

His research projects have been funded by Italian Ministries, by the Italian National Research Council, by major companies (e.g., Ericsson, Telecom Italia), by the ESA and by the EU.

$\mathrm{He}$ has participated in about $30 \mathrm{EU}$ projects, playing the role of project coordinator for six of them. He has been an elected member of the 5G Public Private Partnership association (https://5g-ppp.eu/), a 1.4 Billion Euro initiative established to create the next generation of networks. He evaluated many research proposals and projects in EU programs and served as TPC member, TPC Chair, General Chair and Steering Committee Chair for IEEE Conferences and guest editor for IEEE Journals. He is an area editor for Elseviers Computer Networks. He is author/co-author of about 200 papers. His research interests lie in the performance evaluation, design and control of telecommunications networks.

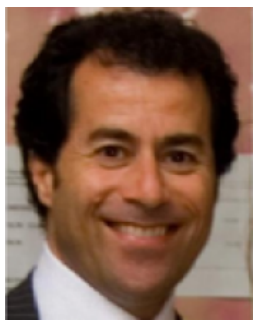

Paolo Dell'Olmo is full professor in Operations Research at the Department of Statistical Sciences (DSS) of Sapienza University of Roma (Italy). Department Head (2005-2011), Coordinator of the $\mathrm{PhD}$ program in Operations Research (2005-2011), President of the Italian Inter-University Center for Operations Research (2010-2015), he is currently Director of the Master Program in Data Intelligence and Strategic Decisions. His research interests are mainly in combinatorial optimization also applied to real life networks problems. In particular: computational complexity, design and analysis of exact and approximated algorithms, mathematical programming applied to traffic management, logistics, coordination of traffic flows on networks, scheduling and routing. He has been scientific coordinator of a number of national research projects and hes author of several books and papers published on international journals.

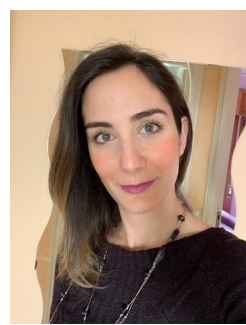

Lavinia Amorosi is researcher at the Department of Statistical Sciences of Sapienza, University of Rome. She received the Ph.D in Operational Research from Sapienza University of Rome in 2018. From November 2017 to March 2018 she worked as researcher at CNIT (Consorzio Nazionale Interuniversitario delle Telecomunicazioni) for the Superfluidity H2020 project. She has been visiting Ph.D at Lancaster University Management School, Lancaster, UK, under the supervision of Prof. Matthias Ehrgott in 2016 and at University of Seville, Spain, under the supervision of Prof. Justo Puerto in 2017. Her research area is mainly on combinatorial optimization, with particular interest in network optimization and multi-objective programming, with application to real network problems in telecommunication and transportation areas.
Antonio Lo Mastro got his Bachelor thesis in ICT and Internet Engineering from the University of Rome Tor Vergata in 2018. He is currently a master thesis student at the University of Rome Sapienza. 


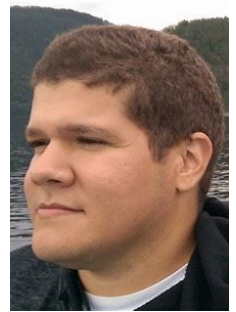

Carlos Natalino (carlos.natalino@chalmers.se) is a Post-Doc Researcher with the Chalmers University of Technology. He received MSc and PhD degree in electrical engineering from Federal University of Par, Brazil in 2011 and 2016, respectively. He was previously a Post-Doc with KTH Royal Institute of Technology, where he spent one year as a visiting researcher during his $\mathrm{PhD}$ (2013-2014). He authored/co-authored more than 40 papers published in international conferences and journals. $\mathrm{He}$ has served as TPC member and reviewer in several international conferences and journals. His research interests are in the fields of optimization and cognitive operation of communication networks, focusing on the dimensioning and operation of survivable multi-domain networks, and on the cognitive operation of optical networks. Carlos Natalino is a Member of IEEE.

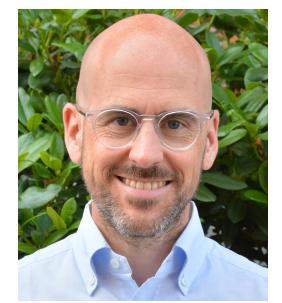

Paolo Monti received a Laurea degree in Electrical Engineering (2001) from the Politecnico di Torino, Italy, and a Ph.D. in Electrical Engineering (2005) from the University of Texas at Dallas (UTD). From 2008 to 2018 he was with KTH Royal Institute of Technology as an Associate Professor in the School of Electrical Engineering and Computer Science (EECS) and the Program Director (20152018) of the Bachelor of Science in Information and Communication Technology (TCOMK). Since 2019, Dr. Monti is with the Department of Electrical Engineering at Chalmers University of Technology as a Professor in Optical Networks where he is also leading the Optical Networks Research Unit.

He published more than 150 papers in peer-reviewed international journals and conferences. Dr. Monti is serving on the editorial board of the IEEE Transactions on Green Communications and Networking, of the IEEE Communications Letters, and of the IEEE Networking Letters journals. He has also been serving as lead and/or co-lead Guest Editor of a number of special issues focused on optical network design, energy efficiency, and resiliency in IEEE, Elsevier, and Springer journals. Dr. Monti regularly participates in the TPCs of the major IEEE and OSA conferences including OFC, IEEE Globecom, and IEEE ICC. He has been the ONS Symposium Chair at the IEEE Globecom 2017, and the TPC chair of IEEE HPSR 2017, IEEE OnlineGreenComm 2016, and ONDM 2014.

His current research interests are within the architectural, technological, programmability, and sustainability challenges of $5 \mathrm{G}$ network infrastructures. Dr. Monti is a Senior Member of IEEE. 